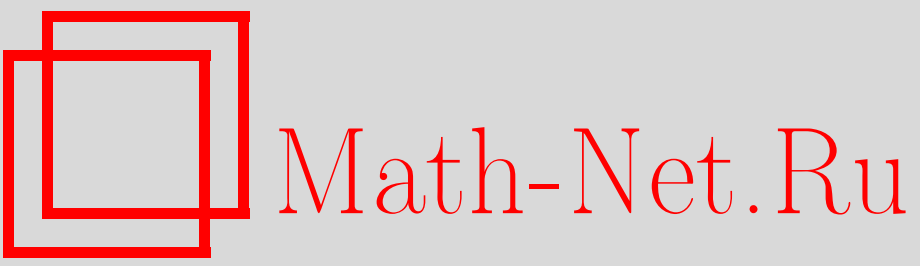

А. К. Погребков, Бозон-фермионное соответствие и квантовые интегрируемые и бездисперсные модели, УМН, 2003, том 58, выпуск 5, 163-196

DOI: https://doi.org/10.4213/rm668

Использование Общероссийского математического портала Math-Net.Ru подразумевает, что вы прочитали и согласны с пользовательским соглашением

http://www.mathnet.ru/rus/agreement

Параметры загрузки:

IP: 54.237 .59 .107

26 апреля 2023 г., 16:38:04 


\section{БОЗОН-ФЕРМИОННОЕ СООТВЕТСТВИЕ И КВАНТОВЫЕ ИНТЕГРИРУЕМЫЕ И БЕЗДИСПЕРСНЫЕ МОДЕЛИ}

\section{А. К. ПОГРЕБКОВ}

Работа посвящена деталному описанию введенного Колеманом и Мандельштамом понятия бозон-фермионного соответствия и приложениям этого соответствия к интегрируемым и связанным с ними моделям. Дана явная формулировка этого соответствия в терминах безмассовых фермионных полей и исследованы свойства полученного скалярного поля. Показано, что это поле является хорошо определенной операторнозначной обобщенной функцией в фермионном фоковском пространстве. В то же время оно не вейлево, а его корреляционные функции не существуют. Далее, реализуя бозонное поле как ток безмассовых (киральных) фермионов, мы выводим иерархию квантовых полиномиальных самодействий этого поля, определяемую условием, что соответствующие эволюционные уравнения фермионных полей линейны. Мы доказываем, что все уравнения этой иерархии вполне интегрируемы, допускают единственные глобалњные решения, однако в классическом пределе эта иерархия сводится к бездисперсной иерархии $\mathrm{K}$ д $\Phi$. Применение нашего построения к квантованию общих вполне интегрируемых взаимодействий продемонстрировано на примере уравнений $\mathrm{K} д \Phi$ и мКд $\Phi$, для которых проводится процедура квантования скобки Гарднера-Захарова-Фаддеева. Показано, что в обоих случаях соответствующие гамильтонианы суть суммы двух хорошо определенных операторов, каждьй из которых билинеен и диагонален по отношению либо к фермионным, либо к бозонным (токовым) операторам рождения-уничтожения. Как результат, процедура квантования не требует никаких пространственных обрезаний и может быть выполнена на всей оси пространственной переменной. Показано, что в рамках нашего подхода в гильбертовом пространстве существуют солитонные состояния, а солитонные параметры квантуются.

Библиография: 46 названий.

\section{СОДЕРЖАНИЕ}

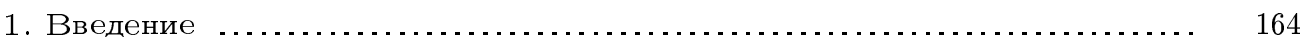

2. Безмассовые двумерные фермионные поля $\ldots \ldots \ldots \ldots \ldots \ldots \ldots \ldots \ldots \ldots \ldots . . \ldots \ldots \ldots$

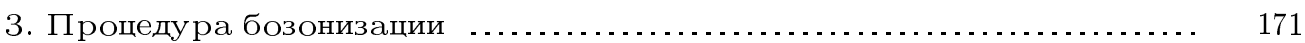

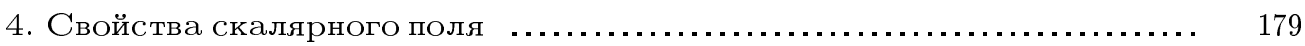

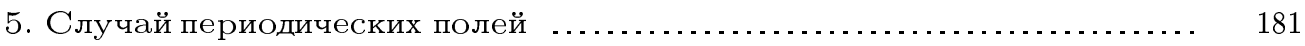

6. Иерархия интегрируемых и явно решаемых моделей $\ldots . \ldots \ldots \ldots \ldots \ldots . . .183$

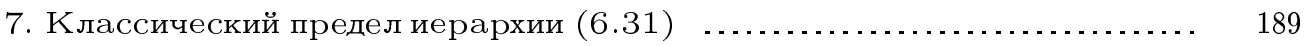

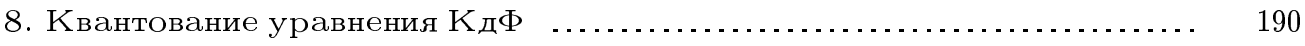

9. Квантование модифицированного уравнения КдФ ................. 192

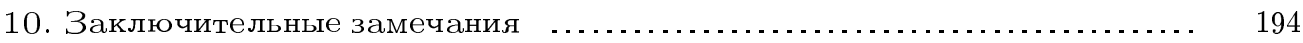

Список литературы ................................................. 195 


\section{1. Введение}

Специальные квантовые поля, которые впервые появились в литературе под названием "безмассовые двумерные фермионные поля" (см., например, [1]) уже в течение десятилетий известны как полезный инструмент исследования вполне интегрируемых моделей в квантовом (соответствие Колемана-Мандельштама, или процедура фермионизашии, [2]-[6]) и в классическом (симметрийньй подход к иерархии Кадомцева-Петвиашвили [7], [8]) случаях. Понятие фермионизации возникло в контексте квантования знаменитого уравнения sine-Гордон около тридцати лет назад [2], [3]. В литературе (см., например, [4], [5], [9]-[12] и приведенные там ссылки) сушествуют различные реализации этой идеи и различные интерпретации результатов. Вопреки первоначальным результатам соответствие Колемана-Мандельштама модели sine-Гордон и массивной модели Тирринга не привело к каким-либо результатам ни для одной из этих моделей. Тем не менее основное наблюдение работ [2], [3] того, что наиболее нелинейные части квантовых бозонных гамильтонианов становятся билинейньми, когда бозонное поле рассматривается как композиция фермионных полей, было подтверждено другими примерами. Так, в [13]-[15] это же свойство было доказано для нелинейного уравнения Шрёдингера и некоторых интегрируемых моделей статистической физики, где фермионные поля естественно возникли в так назьваемом пределе бесконечной связи, т.е. снова как описьвающие наиболее нелинейную часть гамильтониана. В [6] такой же результат был доказан для уравнения Кортевега-де $\Phi$ риза (КдФ). В последние годы интерес к процедуре бозонизации вновь возник в связи с развитием конформной квантовой теории поля (см., например, [16]-[22] и приведенные там ссылки).

Исследованию бозон-фермионного соответствия в том виде, как оно в основном представлено в литературе, более подходит название “фермионизация", когда в качестве основного объекта выбирается свободное безмассовое двумерное скалярное поле, а фермионные поля строятся как экспоненты от него. Хорошо известно, что такое поле не сушествует в вайтмановском смысле [1], так что сначала следует отказаться от каких-либо из стандартных свойств (т.е. самосопряженность, лоренщева инвариантность, положительная определенность, неприводимость и т. д.) скалярных полей. Конечно, в таком случае свойства построенных фермионных полей определяются этим первьм шагом и могут быть далекими от ожидающихся.

Подход данной работы основан на хорошо определенном объекте: безмассовом (каноническом) фермионном поле (см. раздел 2, где даны некоторые стандартные определения). Специфическое свойство такого поля состоит в том, что его разложение в смысле операторов рождения и уничтожения совпадает с разложением на части, которые допускают аналитическое продолжение в верхнюю или нижнюю полуплоскости пространственной переменной. Это свойство позволяет интерпретировать бозон-фермионное соответствие как соотношение между аддитивной и мультипликативной задачами Римана-Гильберта для фермионных полей. В разделе 3 статьи мы рассматриваем процедуру “бозонизации”, т.е. мы явно строим бозонные операторы через посредство фермионных. Наша процедура, в частности, естественно включает в себя

Работа выполнена при частичной поддержке Российского фонда фундаментальных исследований (грант № 02-01-00484). 
сплетаюшие операторы, которые в этом контексте впервые появились в [4]. В разделе 4 мы строим скалярное поле и исследуем его свойства. Мы показьваем, что это поле, будучи хорошо определенной операторнозначной обобщенной функцией в гильбертовом пространстве, не вейлево, а вакуумные ожидания произведений этих полей (функции Вайтмана или Грина) не существуют. Рассмотрение разделов 2-4 вьполнено для полей, заданных на всей оси. В разделе 5 мы кратко рассматриваем редукцию к случаю $2 \pi$-периодических полей, ср. [23]. Наше построение здесь основано на свободных фермионных полях, хотя обобщение на такие взаимодействия, как модели Тирринга и Швингера, достаточно прямолинейно по аналогии с [24], [25]. Мы также опускаем здесь рассмотрение анионных полей $[26]$, [27], которые могут дать интересные обобщения предложенной процедуре.

В разделах 6,8 и 9 мы показываем применимость бозон-фермионного соответствия к квантованию уравнения $\mathrm{K} д \Phi$, уравнений иерархии $\mathrm{K} д \Phi$, и уравнений, непосредственно связанных с ними. Знаменитое уравнение Кд $\Phi$

$$
v_{t}=6 v v_{x}-v_{x x x}
$$

на вешественную функцию $v(t, x)$ дало первьй пример вполне интегрируемого дифференциального уравнения [28], т.е. уравнения, которое может быть записано в лаксовой форме как условие совместности двух линейных уравнений

$$
\begin{gathered}
-\varphi_{x x}(x, k)+v(x) \varphi(x, k)=k^{2} \varphi(x, k), \\
\varphi_{t}=-4 \varphi_{x x x}+6 v(x) \varphi_{x}+3 v_{x}(x) \varphi-4 i k^{3} \varphi
\end{gathered}
$$

на вспомогательную функцию $\varphi(x, k)$ (мыне указываем $t$-зависимость во всех тех случаях, когда это не сушественно). Если $v(x)$ - гладкая, вещественная функция, которая достаточно быстро спадает при $|x| \rightarrow \infty$, то к уравнению (1.1) применим метод обратной задачи рассеяния (МОЗР) (см. [29], [30] и приведенные там ссылки). Этот метод основан на прямой и обратной задачах для функции $\varphi(x, k)$ и приводит к линейной эволюции для соответствуюших спектральных данных и к однозначной разрешимости начальной задачи для уравнения (1.1).

Уравнение КдФ было также первым уравнением, для которого была доказана бигамильтоновость. Точнее, существуют две (локальные) пуассоновы структуры: скобка Гарднера-Захарова-Фаддеева, [31], [32],

$$
\{v(x), v(y)\}_{1}=\delta^{\prime}(x-y),
$$

и скобка Магри, [33],

$$
\{v(x), v(y)\}_{2}=(v(x)+v(y)) \delta^{\prime}(x-y)-\frac{\alpha^{2}}{2} \delta^{\prime \prime \prime}(x-y),
$$

такие, что уравнение (1.1) может быть записано в одной из двух форм:

$$
v_{t}=-\left\{H_{\mathrm{KdV}}, v\right\}_{1}, \quad v_{t}=-\left\{H_{\mathrm{KdV}}^{\prime}, v\right\}_{2},
$$

где гамильтонианы $H_{1}$ и $H_{2}$ суть

$$
\begin{aligned}
& H_{\mathrm{KdV}}=\int d x\left(v^{3}(x)+\frac{\alpha^{2}}{2} v_{x}^{2}(x)\right), \\
& H_{\mathrm{KdV}}^{\prime}=\int d x v^{2}(x) .
\end{aligned}
$$


Квантование уравнения Кд $\Phi$ в периодическом случае было существенно для конформной теории поля [34]-[37], поскольку пуассоновы структуры (1.4) и (1.5) совпадают с соответствуюшими коммутационными соотношениями для алгебры токов и алгебры Вирасоро. Стандартное квантование теории на всей оси требует некоторой регуляризации (например, пространственного обрезания) гамильтониана, чтобы придать ему соответствующий смысл оператора. Любая такая регуляризация несовместна с МОЗР уже в классическом случае: непрерьвньй и дискретньй спектры задачи (1.2) перепутьваются и наиболее интересные, солитонные, решения исчезают. В [6] было показано, что квантование каждой из указанных скобок для уравнения КдФ может быть выполнено посредством процедуры фермионизации на всей $x$-оси и соответствующие квантовые гамильтонианы даются суммой двух членов, билинейных по отношению к либо фермионньм, либо токовым операторам, так что нет необходимости ни в какой процедуре обрезания. Также было доказано, что квантовое бездисперсное уравнение КдФ порождает линейное эволюционное уравнение для ферми-поля. Это подтверждает отмеченное выше наблюдение, что безмассовые фермионы линеаризуют наиболее нелинейную часть бозонных интегрируемых уравнений.

Соответственно, в разделе 6 статьи мы обрашаемся к следуюшей проблеме. Пусть $v(x)$ - квантовое бозонное поле, которое удовлетворяет квантовой версии скобки (1.4). Каковы нелинейные самодействия такого поля, которые при реализации $v$ в качестве тока фермионного поля $\psi$ приводят к линейньм эволюциям для $\psi$ ? Мы доказываем, что это условие однозначно определяет иерархию таких бозонных взаимодействий, которая обладает следующими свойствами:

- все уравнения этой иерархии вполне интегрируемы, в том смысле, что они обладают бесконечным набором локальных, полиномиальных (по отношению к $v$ и его производным), коммутируюших интегралов движения;

- в пределе $\hbar \rightarrow 0$ эта иерархия сводится к бездисперсной иерархии $\mathrm{K} д \Phi$;

- все уравнения этой иерархии однозначно, глобально и явно разрешимы в терминах $v$.

Последнее свойство является специфическим квантовым в противоположность классическому случаю, где бездисперсные уравнения КдФ (и другие уравнения бездисперсной иерархии КдФ) требуют отдельного рассмотрения. В этом случае (см. [29]) МОЗР не применим и бездисперсные уравнения приобретают переворачиваюшиеся решения, т.е. решения, которые не есть однозначно определенные функции $x$. Эти уравнения привлекают внимание в литературе (см., например, [35], [38]-[40]) в контексте теории струн. В разделе 7 мы рассматриваем классический предел иерархии, введенной в разделе 6.

В разделах 8, 9 мы показьваем посредством уравнений КдФ и модифицированного КдФ (мКдФ) применимость бозон-фермионного соответствия к квантованию общих интегрируемых моделей. В частности, мы показьваем, что в гильбертовом пространстве сушествует состояние, которое может быть названо "одно-солитонньм", и что солитонная переменная действия оказьвается квантованной. Некоторые заключительные замечания и обсуждения даны в разделе 10. 


\section{2. Безмассовые двумерные фермионные поля}

Здесь мы вводим обозначения и перечисляем некоторые стандартные свойства безмассовых ферми-полей (см., например, [1]). Пусть $\mathscr{H}$ означает фермионное фоковское пространство по отношению к операторам уничтожения $a(k)$ и $b(k)$ и операторам рождения $a^{*}(k)$ и $b^{*}(k)$, где $k \in \mathbb{R}$ и * означает эрмитово сопряжение. Эти операторы удовлетворяют каноническим антикоммутационным соотношениям

$$
\left[a(k), a^{*}(p)\right]_{+}=\delta(k-p), \quad\left[b(k), b^{*}(p)\right]_{+}=\delta(k-p)
$$

с другими антикоммутаторами, равньми нулю. Пусть $\Omega \in \mathscr{H}$ означает вакуумньй вектор,

$$
a(k) \Omega=0, \quad b(k) \Omega=0,
$$

и $\Omega$ цикличен в $\mathscr{H}$. Двухкомпонентное (правое/левое) безмассовое фермионное поле определяется как фурье-преобразование

$$
\psi_{ \pm}(x)=\varepsilon \int d k e^{i k x}\left[\theta(\mp k) a^{*}(-k)+\theta( \pm k) b(k)\right]
$$

где мы ввели для краткости константу

$$
\varepsilon=\sqrt{\frac{\hbar}{2 \pi}} .
$$

Мы сохраняем постоянную Планка $\hbar$ в соотношениях типа (2.3), поскольку в разделе 7 рассматривается классический предел. Поле (2.3) удовлетворяет каноническим антикоммутационньм соотношениям

$$
\begin{gathered}
{\left[\psi_{ \pm}^{*}(x), \psi_{ \pm}(y)\right]_{+}=\hbar \delta(x-y),} \\
{\left[\psi_{ \pm}(x), \psi_{ \pm}(y)\right]_{+}=0, \quad\left[\psi_{ \pm}(x), \psi_{\mp}(y)\right]_{+}=0,}
\end{gathered}
$$

и имеет следуюшие вакуумные ожидания:

$$
\left(\Omega, \psi_{ \pm}(x) \psi_{ \pm}^{*}(y) \Omega\right)=\left(\Omega, \psi_{ \pm}^{*}(x) \psi_{ \pm}(y) \Omega\right)=\frac{ \pm i \varepsilon^{2}}{x-y \pm i 0}
$$

В силу свободной временно́й эволюции введенное поле развивается как

$$
\psi_{ \pm}(t, x)=\psi_{ \pm}(x \mp t)
$$

так что оно удовлетворяет безмассовому двумерному уравнению Дирака, что дало название этому полю, хотя, как мы отмечали во введении, его область приложений сушественно шире. Соответствуюший гамильтониан, порождающий эту временную эволюцию (2.7), и оператор импульса даются как

$$
\begin{aligned}
& H=H_{+}+H_{-}=\int d k|k|\left[b^{*}(k) b(k)+a^{*}(k) a(k)\right], \\
& P=H_{+}-H_{-}=\int d k k\left[b^{*}(k) b(k)+a^{*}(k) a(k)\right],
\end{aligned}
$$


где

$$
H_{ \pm}=\int d k|k| \theta( \pm k)\left[b^{*}(k) b(k)+a^{*}(k) a(k)\right]
$$

В описании двумерных безмассовых фермионных полей критическую роль играют правые/левые токи, задаваемые как билинейные комбинации

$$
v_{ \pm}(x)=\varepsilon^{-1}: \psi_{ \pm}^{*} \psi_{ \pm}:(x),
$$

где нижний индекс $x$ означает производную по $x$, а знак : . . : означает виковское упорядочение по отношению к фермионньм операторам рождения-уничтожения, например,

$$
: \psi_{ \pm}^{*}(x) \psi_{ \pm}(y):=\psi_{ \pm}^{*}(x) \psi_{ \pm}(y)-\left(\Omega, \psi_{ \pm}^{*}(x) \psi_{ \pm}(x) \Omega\right)
$$

и

$$
: \psi_{ \pm}^{*} \psi_{ \pm}:(x)=\lim _{y \rightarrow x}: \psi_{ \pm}^{*}(x) \psi_{ \pm}(y):
$$

и т. д. Токи являются самосопряженными операторнозначньми обобщенными функциями в пространстве $\mathscr{H}$, удовлетворяющими следуюшим коммутационньм соотношениям:

$$
\begin{aligned}
{\left[\psi_{ \pm}(x), v_{ \pm}(y)\right] } & =2 \pi \varepsilon \delta(x-y) \psi(x) \\
{\left[v_{ \pm}(x), v_{ \pm}(y)\right] } & =\mp i \hbar \delta^{\prime}(x-y)
\end{aligned}
$$

в то время как коммутаторы полей с противоположньми киральностями равны нулю. Заряды фермионного поля определены посредством

$$
\Lambda_{ \pm}=\frac{1}{2 \pi} \int d x v_{ \pm}(x)
$$

они являются самосопряженными операторами, которые в силу (2.3) в терминах операторов рождения-уничтожения имеют представление

$$
\Lambda_{ \pm}=\varepsilon \int_{0}^{\infty} d k\left(b^{*}( \pm k) b( \pm k)-a^{*}( \pm k) a( \pm k)\right)
$$

так что их спектры равны $\varepsilon \mathbb{Z}$. Эти операторы зануляют вакуум,

$$
\Lambda_{ \pm} \Omega=0
$$

и удовлетворяют коммутационным соотношениям

$$
\left[\Lambda_{ \pm}, \psi_{ \pm}(x)\right]=-\varepsilon \psi_{ \pm}(x)
$$

Коммутационное соотношение (2.15) предлагает интерпретацию $v(x)$ как бозонного поля, которое удовлетворяет квантованной версии скобки (1.4), см. разделы 6-9 ниже.

В дальнейшем мы используем для произвольной функции $f(x)$ (операторнозначной обобщенной функции) следующее разложение на положительную и отрищательную части:

$$
f(x)=f^{+}(x)+f^{-}(x)
$$


где

$$
f^{ \pm}(x)=\frac{ \pm 1}{2 \pi i} \int \frac{d y f(y)}{y-x \mp i 0},
$$

так что они допускают аналитическое продолжение в верхнюю и нижнюю полуплоскости переменной $x$ соответственно. Комплексное (или эрмитово) сопряжение обрашает определение этих компонент:

$$
\left(f^{ \pm}\right)^{*}=f^{* \mp}
$$

Во введении уже отмечалось, что специфическим свойством безмассовых фермионов является то, что это разложение для полей и их токов совпадает с разложением в смысле операторов рождения-уничтожения. Так, по (2.3) мы получаем, что операторы $\psi_{ \pm}^{ \pm}$и $\psi_{ \pm}^{*}{ }^{ \pm}$зануляют вакуум, а $\psi_{ \pm}^{\mp}$ и $\psi_{ \pm}^{*}$ являются операторами рождения, с ненулевьми антикоммутаторами, равньми

$$
\left[\psi_{ \pm}^{*} \sigma(x), \psi_{ \pm}^{-\sigma}(y)\right]_{+}=\frac{i \sigma \varepsilon^{2}}{x-y+i \sigma 0}, \quad \sigma=+,-
$$

Аналогично, для токов мы имеем:

$$
v_{ \pm}^{ \pm}(x) \Omega=0
$$

a $v_{ \pm}^{\mp}$ являются операторами рождения. Принимая во внимание, что $v(x)$ - самосопряженный оператор, мы получаем по (2.22), что

$$
\left(v_{ \pm}^{ \pm}(x)\right)^{*}=v_{ \pm}^{\mp}(x) .
$$

Итак, $v_{ \pm}^{\mp}$ и $v_{ \pm}^{ \pm}$являются бозонньми операторами рождения и уничтожения соответственно, которые билинейны по отношению к фермионным. Следуя [1], можно ввести бозонное виковское упорядочение, которое мы обозначаем символом $\vdots . .$. и которое означает, что все бозонные операторы рождения помешаются слева от операторов уничтожения, например,

$$
\vdots v_{ \pm}(x) v_{ \pm}(y) \vdots=v_{ \pm}^{\mp}(x) v_{ \pm}^{\mp}(y)+v_{ \pm}^{\mp}(x) v_{ \pm}^{ \pm}(y)+v_{ \pm}^{\mp}(y) v_{ \pm}^{ \pm}(x)+v_{ \pm}^{ \pm}(x) v_{ \pm}^{ \pm}(y)
$$

и снова

$$
\vdots v_{ \pm}^{2} \vdots(x)=\lim _{y \rightarrow x} \vdots v_{ \pm}(x) v_{ \pm}(y) \vdots
$$

Можно также использовать равенство

$$
\vdots v_{ \pm}(x) v_{ \pm}(y) \vdots=v_{ \pm}(x) v_{ \pm}(y)-\left(\Omega, v_{ \pm}(x) v_{ \pm}(y) \Omega\right)
$$

где

$$
\left(\Omega, v_{ \pm}(x) v_{ \pm}(y) \Omega\right)=\left(\frac{i \varepsilon}{x-y \pm i 0}\right)^{2}
$$


Процедура фермионизации основана на связи между этими фермионным и бозонным нормальными упорядочениями. Например, используя в правой части равенства (2.28) определение (2.11) и теорему Вика для фермионных операторов, получаем

$$
\vdots v_{ \pm}(x) v_{ \pm}(y):=: v_{ \pm}(x) v_{ \pm}(y): \pm i \frac{: \psi_{ \pm}^{*}(x) \psi_{ \pm}(y):-: \psi_{ \pm}^{*}(y) \psi_{ \pm}(x):}{x-y}
$$

В пределе $y \rightarrow x$ мы используем свойство фермионов: любое выражение типа $: \cdots \psi(x) \cdots \psi(x) \cdots$ : обрашается в нуль. Это сушественно понижает степени фермионных полей и упрошает результаты. Например, в (2.30) первьй член в правой части исчезает в этом пределе, и мы получаем

$$
\vdots v_{ \pm}^{2}:(x)=\mp i\left(: \psi_{ \pm}^{*} \psi_{ \pm, x}:(x)-: \psi_{ \pm, x}^{*} \psi^{ \pm}:(x)\right)
$$

Соотношения такого типа существуют также для более высоких степеней,

$$
\vdots v_{ \pm}^{3} \vdots(x)=3 \varepsilon: \psi_{ \pm, x}^{*} \psi_{ \pm, x}:(x)-\frac{\varepsilon^{2}}{2} v_{ \pm, x x}(x)
$$

где снова вьпали старшие степени фермионных операторов. Уравнения (2.30) также можно использовать для вьвода соотношений между производными токов. Например,

$$
\begin{aligned}
\vdots v_{ \pm, x}^{2} \vdots(x)=: v_{ \pm, x}^{2}:(x) \pm \frac{i}{6}\left(: \psi_{ \pm, x x x}^{*} \psi_{ \pm}:(x)-: \psi_{ \pm, x x}^{*} \psi_{ \pm, x}:(x)\right. \\
\left.+: \psi_{ \pm, x}^{*} \psi_{ \pm, x x}:(x)-: \psi_{ \pm}^{*} \psi_{ \pm, x x x}:(x)\right),
\end{aligned}
$$

где $: v_{ \pm, x}^{2}:=2 \varepsilon^{-2}: \psi_{ \pm, x}^{*} \psi_{ \pm}^{*} \psi_{ \pm, x} \psi_{ \pm}:$, и т. п.

Бозонное упорядочение $\vdots . . . \vdots$ можно расширить [24] на выражения, содержашие фермионные поля:

$$
\vdots v_{ \pm}(x) \psi_{ \pm}(y) \vdots=v_{ \pm}^{\mp}(x) \psi_{ \pm}(y)+\psi_{ \pm}(y) v_{ \pm}^{ \pm}(x)
$$

Тогда по (2.14) и $(2.21)$

$$
\vdots v_{ \pm}(x) \psi_{ \pm}(y) \vdots=: v_{ \pm}(x) \psi_{ \pm}(y): \mp i \varepsilon \frac{\psi_{ \pm}(x)-\psi_{ \pm}(y)}{x-y}
$$

так что это выражение, равно как и его производные по $x$ и $y$, хорошо определено в пределе $y \rightarrow x$, и мы получаем

$$
\vdots \frac{\partial^{n} v_{ \pm}}{\partial x^{n}} \psi_{ \pm} \vdots(x)=: \frac{\partial^{n} v_{ \pm}}{\partial x^{n}} \psi_{ \pm}:(x) \mp \frac{i \varepsilon}{(n+1)} \frac{\partial^{n+1} \psi_{ \pm}(x)}{\partial x^{n+1}}, \quad n=0,1,2, \ldots
$$

Используя то же самое свойство фермионных операторов, что и при вьводе равенства (2.31), мы при $n=0$ получаем соотношение

$$
\vdots_{ \pm} \psi_{ \pm} \vdots(x)=\mp i \varepsilon \psi_{ \pm, x}(x)
$$


которое приводит к бозонизации фермионов [2]-[5]. Действительно, это уравнение для $\psi_{ \pm}$можно (но крайней мере, формально) проинтегрировать, что дает

$$
\psi_{ \pm}(x)=\vdots e^{ \pm i \varepsilon^{-1} \int^{x} v_{ \pm}(x) d x}: \equiv e^{ \pm i \varepsilon^{-1} \int^{x} v_{ \pm}^{\mp}(x) d x} e^{ \pm i \varepsilon^{-1} \int^{x} v_{ \pm}^{ \pm}(x) d x}
$$

где во втором равенстве мы воспользовались определением бозонного нормального упорядочения для экспонент. Соотношение (2.38) требует специальной инфракрасной регуляризации первообразной тока, $\int^{x} v_{ \pm}(x) d x$, что будет проделано в следуюшем разделе.

\section{3. Процедура бозонизации}

Теперь мы придадим точньй математический смысл процедуре бозонизации [2], [3], [41] для фермионов, т.е. мы конкретизируем равенство (2.38). Принимая во внимание, что операторы $v_{ \pm}^{ \pm}(x)$ и $v_{\mp}^{ \pm}(x)$ допускают аналитическое продолжение в верхнюю/нижнюю полуплоскости переменной $x$, мы в дальнейшем используем полужирные обозначения для комплексных переменных, $\mathbf{x}=\mathbf{x}_{\operatorname{Re}}+i \mathbf{x}_{\operatorname{Im}} \in \mathbb{C}$, сохраняя стандартные обозначения для их вещественных частей:

$$
x=\mathbf{x}_{\mathrm{Re}}, \text { и т.п. }
$$

Пусть также

$$
\mathbb{C}_{ \pm}=\{\mathbf{x} \in \mathbb{C}, \pm \mathbf{x}>0\}, \quad \overline{\mathbb{C}}_{ \pm}=\{\mathbf{x} \in \mathbb{C}, \pm \mathbf{x} \geqslant 0\}
$$

Мы вводим первообразные токов посредством соотношений

$$
\begin{aligned}
& V_{ \pm}^{ \pm}(\mathbf{x}, \mathbf{z})=\int_{\mathbf{z}}^{\mathbf{x}} d \mathbf{y} v_{ \pm}^{ \pm}(\mathbf{y}), \quad \mathbf{x}, \mathbf{z} \in \overline{\mathbb{C}}_{ \pm}, \\
& V_{ \pm}^{\mp}(\overline{\mathbf{x}}, \overline{\mathbf{z}})=\int_{\overline{\mathbf{z}}}^{\overline{\mathbf{x}}} d \overline{\mathbf{y}} v_{ \pm}^{\mp}(\overline{\mathbf{y}}), \quad \mathbf{x}, \mathbf{z} \in \overline{\mathbb{C}}_{ \pm},
\end{aligned}
$$

где $\overline{\mathbf{x}}$ означает комплексное сопряжение и контуры интегрирования по у и $\overline{\mathbf{y}}$ принадлежат соответствуюшим полуплоскостям. Благодаря (2.21), (2.24) и (2.25) понятно, что

$$
\begin{gathered}
V_{ \pm}^{ \pm}(\mathbf{x}, \mathbf{z})=\frac{ \pm i}{2 \pi} \int d y v_{ \pm}(y) \log \frac{\mathbf{x}-y}{\mathbf{z}-y}, \\
V_{ \pm}^{ \pm}(\mathbf{x}, \mathbf{z}) \Omega=0, \\
V_{ \pm}^{\mp}(\overline{\mathbf{x}}, \overline{\mathbf{z}})=\left(V_{ \pm}^{ \pm}(\mathbf{x}, \mathbf{z})\right)^{*} \\
V_{ \pm}^{ \pm}(\mathbf{z}, \mathbf{x})=-V_{ \pm}^{ \pm}(\mathbf{x}, \mathbf{z})
\end{gathered}
$$

а принимая во внимание, что в силу $(2.29)$

$$
\left(\Omega, V_{ \pm}^{ \pm}(\mathbf{x}, \mathbf{z}) V_{ \pm}^{\mp}\left(\overline{\mathbf{x}}^{\prime}, \overline{\mathbf{z}}^{\prime}\right) \Omega\right)=\varepsilon^{2} \log \frac{\left(\mathbf{x}-\overline{\mathbf{x}}^{\prime}\right)\left(\mathbf{z}-\overline{\mathbf{z}}^{\prime}\right)}{\left(\mathbf{x}-\overline{\mathbf{z}}^{\prime}\right)\left(\mathbf{z}-\overline{\mathbf{x}}^{\prime}\right)}
$$


мы видим, что операторы $V_{ \pm}^{ \pm}(\mathbf{x}, \mathbf{z})$ и $V_{ \pm}^{\mp}(\overline{\mathbf{x}}, \overline{\mathbf{z}})$ плотно определены в $\mathscr{H}$, если мнимые части $\mathbf{x}$ и $\mathbf{z}$ отличны от нуля, $\mathbf{x}, \mathbf{z} \in \mathbb{C}_{ \pm}$.

Свойства экспонент этих операторов даются следуюшими утверждениями.

УТВЕРЖДЕНИЕ 3.1. Пусть $y \in \mathbb{R} u \mathbf{x}, \mathbf{z} \in \mathbb{C}_{ \pm}$. Тогда

$$
\begin{gathered}
e^{ \pm i \varepsilon^{-1} V_{ \pm}^{ \pm}(\mathbf{x}, \mathbf{z})} \psi_{ \pm}^{\sigma}(y) e^{\mp i \varepsilon^{-1} V_{ \pm}^{ \pm}(\mathbf{x}, \mathbf{z})}=\frac{(y-\mathbf{x}) \psi_{ \pm}^{\sigma}(y) \pm \sigma(\mathbf{x}-\mathbf{z}) \psi_{ \pm}^{ \pm}(\mathbf{z})}{y-\mathbf{z}}, \\
e^{ \pm i \varepsilon^{-1} V_{ \pm}^{ \pm}(\mathbf{x}, \mathbf{z})} \psi_{ \pm}^{* \sigma}(y) e^{\mp i \varepsilon^{-1} V_{ \pm}^{ \pm}(\mathbf{x}, \mathbf{z})}=\frac{(y-\mathbf{z}) \psi_{ \pm}^{* \sigma}(y) \mp \sigma(\mathbf{x}-\mathbf{z}) \psi_{ \pm}^{*}(\mathbf{x})}{y-\mathbf{x}},
\end{gathered}
$$

где $\sigma=+,-$. Соотношения для противоположных знаков показателей следуют из (3.8).

ДокАЗАТЕЛЬСтво. Пусть $f(\mathbf{x})$ означает левую часть равенства (3.10). Принимая во внимание, что $\left[V_{ \pm}^{ \pm}, V_{ \pm}^{ \pm}\right]=0$, мы получаем в силу (3.3) для производной по $\mathbf{x}:$

$$
f_{\mathbf{x}}(\mathbf{x})= \pm \frac{i}{\varepsilon} e^{ \pm i \varepsilon^{-1} V_{ \pm}^{ \pm}(\mathbf{x}, \mathbf{z})}\left[v_{ \pm}^{ \pm}(\mathbf{x}), \psi_{ \pm}^{\sigma}(y)\right] e^{\mp i \varepsilon^{-1} V_{ \pm}^{ \pm}(\mathbf{x}, \mathbf{z})}
$$

или по $(2.21)$

$$
f_{\mathbf{x}}(\mathbf{x})=\frac{1}{2 \pi \varepsilon} e^{ \pm i \varepsilon^{-1} V_{ \pm}^{ \pm}(\mathbf{x}, \mathbf{z})} \int \frac{d x^{\prime}}{x^{\prime}-\mathbf{x}}\left[v_{ \pm}\left(x^{\prime}\right), \psi_{ \pm}^{\sigma}(y)\right] e^{\mp i \varepsilon^{-1} V_{ \pm}^{ \pm}(\mathbf{x}, \mathbf{z})} .
$$

Тогда благодаря (2.14) мы приходим к уравнению

$$
f_{\mathbf{x}}(\mathbf{x})=\frac{f(\mathbf{x})}{\mathbf{x}-y}
$$

которое после интегрирования дает (3.10), если принять во внимание, что в силу (3.3) $f(\mathbf{z})=\psi_{ \pm}^{\sigma}(y)$. Равенство (3.11) доказьвается аналогично.

Отметим, что обе части уравнений (3.10) и (3.11) допускают аналитическое продолжение по $y$ в полуплоскости $\overline{\mathbb{C}}_{\sigma}$ и не встречают там препятствий. Действительно, при $\sigma=\mp$ это - продолжение в полуплоскость, противоположную $\mathbb{C}_{ \pm}$, которой принадлежат $\mathbf{x}$ и $\mathbf{y}$, а для $\sigma= \pm$ нуль знаменателя компенсируется нулем числителя.

УТВЕРЖДЕНИЕ 3.2. Для любых $\mathbf{x}, \mathbf{z} \in \mathbb{C}_{ \pm}$экспоненты от $V_{ \pm}^{ \pm}(\mathbf{x}, \mathbf{z})$ даются в терминах операторов, нормально упорядоченных по отношению к фермионам, посредством соотношений

$$
\begin{aligned}
& \exp \left( \pm i \varepsilon^{-1} V_{ \pm}^{ \pm}(\mathbf{x}, \mathbf{z})\right)=:\left\{1 \pm i \frac{\mathbf{x}-\mathbf{z}}{\varepsilon^{2}} \psi_{ \pm}^{*}(\mathbf{z}) \psi_{ \pm}^{ \pm}(\mathbf{x})\right\} \\
& \times \exp \left(\frac{\mathbf{x}-\mathbf{z}}{2 \pi \varepsilon^{2}} \int d x^{\prime}\left[\frac{\psi_{ \pm}^{*}\left(x^{\prime}\right) \psi_{ \pm}^{ \pm}\left(x^{\prime}\right)}{x^{\prime}-\mathbf{x}}+\frac{\psi_{ \pm}^{*}\left(x^{\prime}\right) \psi_{ \pm}^{\mp}\left(x^{\prime}\right)}{x^{\prime}-\mathbf{z}}\right]\right): \\
& \exp \left( \pm i \varepsilon^{-1} V_{ \pm}^{\mp}(\overline{\mathbf{x}}, \overline{\mathbf{z}})\right)=:\left\{1 \pm i \frac{\overline{\mathbf{x}}-\overline{\mathbf{z}}}{\varepsilon^{2}} \psi_{ \pm}^{*}(\overline{\mathbf{z}}) \psi_{ \pm}^{\mp}(\overline{\mathbf{x}})\right\} \\
& \quad \times \exp \left(\frac{\overline{\mathbf{z}}-\overline{\mathbf{x}}}{2 \pi \varepsilon^{2}} \int d x^{\prime}\left[\frac{\psi_{ \pm}^{*}\left(x^{\prime}\right) \psi_{ \pm}^{ \pm}\left(x^{\prime}\right)}{x^{\prime}-\overline{\mathbf{z}}}+\frac{\psi_{ \pm}^{*}\left(x^{\prime}\right) \psi_{ \pm}^{\mp}\left(x^{\prime}\right)}{x^{\prime}-\overline{\mathbf{x}}}\right]\right):
\end{aligned}
$$

и опять выражения для противоположных знаков показателей следуют из (3.8). 
ДокАЗАТЕльство. Пусть $g(\mathbf{x}, \mathbf{z})$ означает нормально (по отношению к фермионам) упорядоченное выражение для экспоненты в левой части (3.12), т.е.

$$
\begin{gathered}
g(\mathbf{x}, \mathbf{z})=e^{ \pm i \varepsilon^{-1} V_{ \pm}^{ \pm}(\mathbf{x}, \mathbf{z})}, \\
: g(\mathbf{x}, \mathbf{z}):=g(\mathbf{x}, \mathbf{z}) .
\end{gathered}
$$

Принимая во внимание, что операторы $v_{ \pm}^{ \pm}$коммутируют сами с собой, мы получаем по $(2.11),(2.21)$ и $(3.3)$ для производной $g(\mathbf{x}, \mathbf{z})$ по $\mathbf{x}$ :

$$
g_{\mathbf{x}}(\mathbf{x}, \mathbf{z})=\frac{-1}{2 \pi \varepsilon} g(\mathbf{x}, \mathbf{z}) \int d y \frac{v_{ \pm}(y)}{\mathbf{x}-y} .
$$

Используя разложение $(2.20)$ для полей $\psi$ и $\psi^{*}$ в определении $v_{ \pm}(2.11)$, мы получаем

$$
v_{ \pm}(y)=\frac{1}{\varepsilon}\left(\psi_{ \pm}^{*}(y) \psi_{ \pm}^{\mp}(y)-\psi_{ \pm}^{\mp}(y) \psi_{ \pm}^{* \pm}(y)+\psi_{ \pm}^{*}{ }^{\mp}(y) \psi_{ \pm}^{ \pm}(y)+\psi_{ \pm}^{*}(y) \psi_{ \pm}^{ \pm}(y)\right),
$$

где фермионное виковское упорядочение проведено явно (ср. обсуждение после (2.22)). Подставляя это выражение в предыдуший интеграл, мы видим, что благодаря свойствам аналитичности объектов, определенных по (2.21), первьй член вьпадает, а второй интегрируется явно, что дает нам

$$
\begin{gathered}
g_{\mathbf{x}}(\mathbf{x}, \mathbf{z})=\frac{1}{2 \pi \varepsilon^{2}} g(\mathbf{x}, \mathbf{z})\left(\int \frac{d y}{y-\mathbf{x}}\left(\psi_{ \pm}^{*}{ }^{\mp}(y) \psi_{ \pm}^{ \pm}(y)-\psi_{ \pm}^{\mp}(y) \psi_{ \pm}^{*}{ }^{ \pm}(y)\right)\right. \\
\left. \pm 2 \pi i \psi_{ \pm}^{*}(\mathbf{x}) \psi_{ \pm}^{ \pm}(\mathbf{x})\right)
\end{gathered}
$$

Слагаемые в подьнтегральном выражении даны как нормально упорядоченные произведения фермионных операторов рождения-уничтожения. Поэтому мы используем утверждение 3.1 , чтобы переместить операторы рождения подынтегрального выражения налево от $g(\mathbf{x}, \mathbf{z})$. Последний член представляет собой произведение двух операторов уничтожения, так что он автоматически дает нормально упорядоченное выражение при умножении слева на $g(\mathbf{x}, \mathbf{z})$. Итак, по $(3.10),(3.11)$ для $\sigma=\mp$ получаем

$$
\begin{aligned}
g_{\mathbf{x}}(\mathbf{x}, \mathbf{z})= & \frac{1}{2 \pi \varepsilon^{2}}\left(\int \frac { d y } { y - \mathbf { x } } \left[\left(\frac{y-\mathbf{z}}{y-\mathbf{x}} \psi_{ \pm}^{*}(y)+\frac{\mathbf{x}-\mathbf{z}}{y-\mathbf{x}} \psi_{ \pm}^{*}(\mathbf{x})\right) g(\mathbf{x}, \mathbf{z}) \psi_{ \pm}^{ \pm}(y)\right.\right. \\
- & \left.\left(\frac{y-\mathbf{x}}{y-\mathbf{z}} \psi_{ \pm}^{\mp}(y)-\frac{\mathbf{x}-\mathbf{z}}{y-\mathbf{z}} \psi_{ \pm}^{ \pm}(\mathbf{z})\right) g(\mathbf{x}, \mathbf{z}) \psi_{ \pm}^{*}(y)\right] \\
& \left. \pm 2 \pi i g(\mathbf{x}, \mathbf{z}) \psi_{ \pm}^{*}(\mathbf{x}) \psi_{ \pm}^{ \pm}(\mathbf{x})\right)
\end{aligned}
$$

Это выражение все еще не является нормально упорядоченным: операторы уничтожения $\psi_{ \pm}^{*}(\mathbf{x})$ и $\psi_{ \pm}^{ \pm}(\mathbf{z})$ все еще находятся слева от $g(\mathbf{x}, \mathbf{z})$. Снова по (3.10), меняя местами переменные $\mathbf{x}$ и $\mathbf{z}$, мы находим для $\sigma= \pm$

$$
\psi_{ \pm}^{ \pm}(y) g(\mathbf{x}, \mathbf{z})=g(\mathbf{x}, \mathbf{z}) \frac{(\mathbf{z}-y) \psi_{ \pm}^{ \pm}(y)+(\mathbf{x}-\mathbf{z}) \psi_{ \pm}^{ \pm}(\mathbf{x})}{\mathbf{x}-y} .
$$


Аналитическое продолжение этого равенства в точку $y=\mathbf{z}$ дает нам

$$
\begin{aligned}
\psi_{ \pm}^{ \pm}(\mathbf{z}) g(\mathbf{x}, \mathbf{z}) & =g(\mathbf{x}, \mathbf{z}) \psi_{ \pm}^{ \pm}(\mathbf{x}), \\
\psi_{ \pm}^{*}(\mathbf{x}) g(\mathbf{x}, \mathbf{z}) & =g(\mathbf{x}, \mathbf{z}) \psi_{ \pm}^{*}(\mathbf{z}),
\end{aligned}
$$

где второе равенство выводится аналогично первому из (3.11). Благодаря этим равенствам уравнение для $g(\mathbf{x}, \mathbf{z})$ становится нормально упорядоченным, и мы можем записать его в виде

$$
\begin{aligned}
g_{\mathbf{x}}(\mathbf{x}, \mathbf{z})= & \frac{1}{2 \pi \varepsilon^{2}}: g(\mathbf{x}, \mathbf{z})\left(\int d y\left(\frac{y-\mathbf{z}}{(y-\mathbf{x})^{2}} \psi_{ \pm}^{*}{ }^{\mp}(y) \psi_{ \pm}^{ \pm}(y)-\frac{y-\mathbf{x}}{y-\mathbf{z}} \psi_{ \pm}^{\mp}(y) \psi_{ \pm}^{*}(y)\right)\right. \\
& +\int d y\left(\frac{\mathbf{x}-\mathbf{z}}{(y-\mathbf{x})^{2}} \psi_{ \pm}^{*}(\mathbf{z}) \psi_{ \pm}^{ \pm}(y)+\frac{\mathbf{x}-\mathbf{z}}{(y-\mathbf{x})(y-\mathbf{z})} \psi_{ \pm}^{ \pm}(\mathbf{x}) \psi_{ \pm}^{*}(y)\right) \\
& \left. \pm 2 \pi i \psi_{ \pm}^{*}(\mathbf{x}) \psi_{ \pm}^{ \pm}(\mathbf{x})\right):
\end{aligned}
$$

Теперь интегрирование во второй строке можно вьполнить явно благодаря свойствам аналитичности, которые следуют из (2.21), так что окончательно получаем

$$
\begin{aligned}
g_{\mathbf{x}}(\mathbf{x}, \mathbf{z})= & : g(\mathbf{x}, \mathbf{z}) \frac{\partial}{\partial \mathbf{x}}\left\{ \pm i \frac{\mathbf{x}-\mathbf{z}}{\varepsilon^{2}} \psi_{ \pm}^{*}(\mathbf{z}) \psi_{ \pm}^{ \pm}(\mathbf{x})\right. \\
& \left.+\frac{\mathbf{x}-\mathbf{z}}{2 \pi \varepsilon^{2}} \int d x^{\prime}\left[\frac{\psi_{ \pm}^{*}\left(x^{\prime}\right) \psi_{ \pm}^{ \pm}\left(x^{\prime}\right)}{x^{\prime}-\mathbf{x}}+\frac{\psi_{ \pm}^{*}\left(x^{\prime}\right) \psi_{ \pm}^{\mp}\left(x^{\prime}\right)}{x^{\prime}-\mathbf{z}}\right]\right\}:
\end{aligned}
$$

что доказьвает (3.12), если принять во внимание, что $g(\mathbf{z}, \mathbf{z}) \equiv 1$ и что в силу свойства фермионов под знаком нормального произведения имеем

$$
: \exp \left(\alpha \psi_{ \pm}^{*}{ }^{ \pm}(\mathbf{z}) \psi_{ \pm}^{ \pm}(\mathbf{x})\right): \equiv 1+\alpha: \psi_{ \pm}^{*}(\mathbf{z}) \psi_{ \pm}^{ \pm}(\mathbf{x}): .
$$

Равенство (3.13) доказьвается аналогично.

Важное свойство равенств (3.12), (3.13) состоит в том, что показатели содержат только члены, пропорциональные произведению оператора рождения на оператор уничтожения.

Легко видеть, что операторы $V_{\mp}^{ \pm}(\mathbf{x}, \mathbf{z})$ и $V_{ \pm}^{ \pm}(\mathbf{x}, \mathbf{z})$, равно как и экспоненты от них, допускают пределы, когда одна или обе из переменных $\mathbf{x}$ и $\mathbf{z}$ стремятся на вешественную ось. Эти пределы определены в смысле операторнозначных обобшенных функций вешественных частей (3.1) этих переменных. Итак, введем

$$
\begin{aligned}
& V_{ \pm}^{ \pm}(x)=V_{ \pm}^{ \pm}(x \pm i 0, \pm i c), \\
& V_{ \pm}^{\mp}(x)=V_{ \pm}^{\mp}(x \mp i 0, \mp i c),
\end{aligned}
$$

где $c$ - некоторый вешественный положительньй параметр. Благодаря (3.6)-(3.9) эти операторнозначные обобщенные функции удовлетворяют

$$
\begin{gathered}
V_{ \pm}^{ \pm}(x) \Omega=0, \quad V_{ \pm}^{\mp}(x)=\left(V_{ \pm}^{ \pm}(x)\right)^{*} \\
{\left[V_{ \pm}^{ \pm}(x), V_{ \pm}^{\mp}\left(x^{\prime}\right)\right]=\varepsilon^{2} \log \frac{(\mp 2 i c)\left(x-x^{\prime} \pm i 0\right)}{(x \pm i c)\left(x^{\prime} \mp i c\right)},}
\end{gathered}
$$


в то время как все другие коммутаторы обращаются в нуль. Для того чтобы записать предельные значения соотношений (3.12), (3.13), удобно ввести постоянные операторы

$$
E_{ \pm}=: \exp \frac{\mp c}{\pi \varepsilon^{2}} \int d x\left(\frac{\psi_{ \pm}^{* \mp}(x) \psi_{ \pm}^{ \pm}(x)}{x \mp i c}-\frac{\psi_{ \pm}^{\mp}(x) \psi_{ \pm}^{* \pm}(x)}{x \pm i c}\right)
$$

$$
R_{ \pm}=a_{ \pm}^{*} E_{ \pm}+E_{ \pm} b_{ \pm}
$$

где мы ввели обозначения для специальных значений $\psi_{ \pm}$в точках $\pm i c$ :

$$
\begin{aligned}
& a_{ \pm}=\frac{\sqrt{2 c}}{\varepsilon} \psi_{ \pm}^{*}( \pm i c), \\
& b_{ \pm}=\frac{\sqrt{2 c}}{\varepsilon} \psi_{ \pm}^{ \pm}( \pm i c) .
\end{aligned}
$$

Благодаря (2.2)-(2.5) и (2.21) легко видеть, что операторы $a_{ \pm}$и $b_{ \pm}$уничтожают вакуум,

$$
a_{ \pm} \Omega=0, \quad b_{ \pm} \Omega=0
$$

так что эрмитово сопряженные операторы $a_{ \pm}^{*}$ и $b_{ \pm}^{*}$ являются операторами рождения. По $(2.23)$

$$
\left[a_{ \pm}, \psi_{ \pm}^{\mp}(x)\right]_{+}=\frac{\mp i \varepsilon \sqrt{2 c}}{x \mp i c}, \quad\left[b_{ \pm}, \psi_{ \pm}^{*}{ }^{\mp}(x)\right]_{+}=\frac{\mp i \varepsilon \sqrt{2 c}}{x \mp i c}
$$

так что

$$
\left[a_{ \pm}^{*}, a_{ \pm}\right]_{+}=1, \quad\left[b_{ \pm}^{*}, b_{ \pm}\right]_{+}=1
$$

с другими антикоммутаторами, равньми нулю.

Теперь соответствие Колемана-Мандельштама можно сфформулировать в виде следуюшей теоремы.

ТЕОрема 3.1. В смысле операторнозначных обобщенных функций мы имеем представление:

$$
\psi_{ \pm}(x)=\frac{\sqrt{2 c}}{\varepsilon} e^{ \pm i \varepsilon^{-1} V_{ \pm}^{\mp}(x)} R_{ \pm} e^{ \pm i \varepsilon^{-1} V_{ \pm}^{ \pm}(x)} .
$$

Мы предпошлем доказательству этой теоремы леммы, описьвающие свойства операторов (3.20) и (3.21).

Лемма 3.1. Операторьи $E_{ \pm}$изометрические:

$$
\begin{aligned}
& E_{ \pm} E_{ \pm}^{*}=1-a_{ \pm}^{*} a_{ \pm}, \\
& E_{ \pm}^{*} E_{ \pm}=1-b_{ \pm}^{*} b_{ \pm},
\end{aligned}
$$

где операторы в правых частях являются ортогональными проекторами. 
ДокАЗАТЕЛЬСтво. Ниже мы регулярно применяем стандартные соотношения, вытекающие из теоремы Вика для экспонент билинейных форм [42]. Пусть $A$ и $B-$ некоторые операторы, билинейные по отношению к $\psi$ и состоящие из мономов вида рождение $\times$ уничтожение. Пусть $a$ - некоторый оператор уничтожения, линейньй по $\psi$. Тогда

$$
: e^{A}:: e^{B}:=: e^{A+B+\underline{A B}}:,
$$

где

$$
\underline{A B}=A B-: A B:
$$

т.е. содержит все члены с одним спариванием в произведении $A B$, и

$$
a: e^{A}:=: e^{A}(a+\underline{a A}):, \quad: e^{A}: a^{*}=:\left(a^{*}+\underline{A a^{*}}\right) e^{A}:
$$

где снова

$$
\underline{a A}=a A-: a A:, \quad \underline{A a^{*}}=A a^{*}-: A a^{*}: .
$$

Выбирая теперь в качестве $A$ показатель экспоненты $E_{ \pm}(3.20)$, а в качестве $B$ его сопряженньй, мы вьводим, что в этом случае

$$
\underline{A B}=\frac{c^{2}}{i \pi \varepsilon^{2}} \int d x \frac{\psi_{ \pm}^{* \mp}(x) \psi_{ \pm}^{ \pm}(x)+\left(\psi_{ \pm}^{\mp}(x)-\psi_{ \pm}^{\mp}(\mp i c)\right) \psi_{ \pm}^{* \pm}(x)}{x^{2}+c^{2}}
$$

где были вьполнены интегрирования в силу аналитических свойств $\psi_{ \pm}^{* \pm}$ и $\psi_{ \pm}^{ \pm}$. Складьвая это выражение с $A+B$ и используя обозначение (3.22), получаем

$$
E_{ \pm} E_{ \pm}^{*}=: \exp \left(-a_{ \pm}^{*} a_{ \pm}\right):
$$

что в свою очередь дает (3.28) в силу фермионных свойств операторов $a_{ \pm}: a_{ \pm}^{2}=0$. Также в силу этого свойства и (3.26) легко доказать, что квадрат правой части (3.28) равен ей самой, в то время как эрмитовость очевидна. Утверждение (3.29) доказывается аналогично.

Лемма 3.2. Для любого $\mathbf{x} \in \mathbb{C}_{ \pm}$имеем следующие соотношения для произведений фермионных полей и операторов $E_{ \pm}$:

$$
\begin{aligned}
\psi_{ \pm}^{ \pm}(\mathbf{x}) E_{ \pm} & =E_{ \pm} \frac{(\mathbf{x} \pm i c) \psi_{ \pm}^{ \pm}(\mathbf{x}) \mp 2 i c \psi_{ \pm}^{ \pm}( \pm i c)}{\mathbf{x} \mp i c}, \\
\psi_{ \pm}^{*}(\mathbf{x}) E_{ \pm} & =\frac{\mathbf{x} \mp i c}{\mathbf{x} \pm i c} E_{ \pm} \psi_{ \pm}^{*}(\mathbf{x}), \\
E_{ \pm} \psi_{ \pm}^{*}(\overline{\mathbf{x}}) & =\frac{\overline{\mathbf{x}} \pm i c}{\overline{\mathbf{x}} i c} \psi_{ \pm}^{*}(\overline{\mathbf{x}}) E_{ \pm}, \\
E_{ \pm} \psi_{\mp}^{ \pm}(\overline{\mathbf{x}}) & =\frac{(\overline{\mathbf{x}} \mp i c) \psi_{ \pm}^{\mp}(\overline{\mathbf{x}}) \pm 2 i c \psi_{ \pm}^{\mp}(\mp i c)}{\mathbf{x} \pm i c} E_{ \pm} .
\end{aligned}
$$


ДОКАЗАТЕЛЬСТвО следует из применения равенства (3.32). Для (3.34) положим $a=\psi_{ \pm}^{ \pm}(\mathbf{x})$ и $A$ равным показателю экспоненты в (3.20). Тогда по (2.23)

$$
\underline{a A}=\frac{c}{\pi} \int d y \frac{\psi_{ \pm}^{ \pm}(y)}{(y-\mathbf{x})(y \mp i c)} .
$$

Благодаря свойству аналитичности функции $\psi_{ \pm}^{ \pm}(y)$ это равенство можно проинтегрировать явно, что дает (3.34). Другие соотношения доказьваются аналогично. Отметим, что по (3.35) и (3.36) мы получаем в силу определений (3.22) и (3.23):

$$
a_{ \pm} E_{ \pm}=0, \quad E_{ \pm} b_{ \pm}^{*}=0 .
$$

Лемма 3.3. Операторы $R_{ \pm}$унитарны,

$$
R_{ \pm}^{*} R_{ \pm}=1=R_{ \pm} R_{ \pm}^{*}
$$

они масштабируют фермионные операторы,

$$
R_{ \pm}^{*} \psi_{ \pm}(x) R_{ \pm}=-\frac{x \pm i c}{x \mp i c} \psi_{ \pm}(x)
$$

и сдвигают токи,

$$
R_{ \pm}^{*} v_{ \pm}(x) R_{ \pm}=v_{ \pm}(x)-\frac{2 \varepsilon c}{x^{2}+c^{2}}
$$

ДоКАЗАТЕЛЬСТво. По (3.21) и (3.26)

$$
R_{ \pm}^{*} R_{ \pm}=E_{ \pm}^{*} E_{ \pm}-E_{ \pm}^{*} a_{ \pm}^{*} a_{ \pm} E_{ \pm}+b_{ \pm}^{*} E_{ \pm}^{*} E_{ \pm} b_{ \pm},
$$

где второй член правой части равен нулю по (3.38). Тогда первое равенство в (3.39) следует в силу (3.29) и идемпотентности, $b^{2}=0$. Второе равенство в (3.39) доказывается аналогично по (3.13).

Для того чтобы доказать (3.40), мы, прежде всего, приведем к нормальной форме произведение $R_{ \pm}^{*} \psi_{ \pm}^{\ddagger}(\overline{\mathbf{x}})$, где $\mathbf{x} \in \overline{\mathbb{C}}_{ \pm}$. По (3.21), применяя последовательно (3.25) и (3.34), мы получаем

$$
R_{ \pm}^{*} \psi_{ \pm}^{\mp}(\overline{\mathbf{x}})=\mp \frac{i \varepsilon \sqrt{2 c} E_{ \pm}^{*}}{\overline{\mathbf{x}} \mp i c}-\frac{\overline{\mathbf{x}} \pm i c}{\overline{\mathbf{x}} \mp i c} \psi_{ \pm}^{\mp}(\overline{\mathbf{x}}) R_{ \pm}^{*} .
$$

Тогда по (3.21) $E_{ \pm}^{*} R_{ \pm}=E_{ \pm}^{*} a_{ \pm}^{*} E_{ \pm}+E_{ \pm}^{*} E_{ \pm} b_{ \pm}$. В силу (3.38) и (3.29) $E_{ \pm}^{*} R_{ \pm}=b_{ \pm}$, так что, принимая во внимание (3.39), мы получаем

$$
R_{ \pm}^{*} \psi_{ \pm}^{\mp}(\overline{\mathbf{x}}) R_{ \pm}=-\frac{\overline{\mathbf{x}} \pm i c}{\overline{\mathbf{x}} \mp i c} \psi_{ \pm}^{\mp}(\overline{\mathbf{x}}) \mp \frac{i \varepsilon \sqrt{2 c}}{\overline{\mathbf{x}} \mp i c} b_{ \pm} .
$$

Аналогично выводится

$$
R_{ \pm}^{*} \psi_{ \pm}^{ \pm}(\mathbf{x}) R_{ \pm}=-\frac{\mathbf{x} \pm i c}{\mathbf{x} \mp i c} \psi_{ \pm}^{ \pm}(\mathbf{x}) \pm \frac{i \varepsilon \sqrt{2 c}}{\mathbf{x} \mp i c} b_{ \pm} .
$$


Теперь суммируя предельные значения предыдущих равенств на вешественной оси $x$, мы доказываем (3.40).

Далее, по (2.6) и (2.12) для вещественных $x$ и $y$ можно написать

$$
R_{ \pm}^{*} \psi_{ \pm}^{*}(y) \psi_{ \pm}(x) R_{ \pm}=\frac{(y \mp i c)(x \pm i c)}{(y \pm i c)(x \mp i c)}\left[: \psi_{ \pm}^{*}(y) \psi_{ \pm}(x): \pm \frac{i \varepsilon^{2}}{y-x \pm i 0}\right]
$$

или

$$
\begin{aligned}
R_{ \pm}^{*}: \psi_{ \pm}^{*}(y) \psi_{ \pm}(x): R_{ \pm}= & \frac{(y \mp i c)(x \pm i c)}{(y \pm i c)(x \mp i c)}: \psi_{ \pm}^{*}(y) \psi_{ \pm}(x): \\
& \pm \frac{i \varepsilon^{2}}{y-x}\left[\frac{(y \mp i c)(x \pm i c)}{(y \pm i c)(x \mp i c)}-1\right]
\end{aligned}
$$

что в пределе $y \rightarrow x$ в силу (2.11) дает (3.41).

ДОКАЗАТЕЛЬСТВО ТЕОРЕМЫ 3.1. Применяя разложение $(2.20),(2.21)$ к равенству (3.41), мы получаем

$$
R_{ \pm}^{*} v_{ \pm}^{ \pm}(x) R_{ \pm}=v_{ \pm}^{ \pm}(x) \mp \frac{i \varepsilon}{x \mp i c}
$$

так что по (3.3) и (3.16)

$$
R_{ \pm}^{*} V_{ \pm}^{ \pm}(x) R_{ \pm}=V_{ \pm}^{ \pm}(x) \mp i \varepsilon \log \frac{c \mp i x}{2 c}
$$

Теперь для правой части (3.27) по (3.39) мы имеем

$$
e^{ \pm i \varepsilon^{-1} V_{ \pm}^{\mp}(x)} R_{ \pm} e^{ \pm i \varepsilon^{-1} V_{ \pm}^{ \pm}(x)}=R_{ \pm} \frac{2 c}{c \pm i x} e^{ \pm i \varepsilon^{-1} V_{ \pm}^{\mp}(x)} e^{ \pm i \varepsilon^{-1} V_{ \pm}^{ \pm}(x)} .
$$

Для произведения последних двух множителей мы воспользуемся соотношением (3.30), что дает

$$
e^{ \pm i \varepsilon^{-1} V_{ \pm}^{\mp}(x)} e^{ \pm i \varepsilon^{-1} V_{ \pm}^{ \pm}(x)}=: \exp \left(\frac{a_{ \pm}-b_{ \pm}^{*}}{\varepsilon \sqrt{2 c}}\left[(c \pm i x) \psi_{ \pm}^{ \pm}(x)-(c \mp i x) \psi_{ \pm}^{\mp}(x)\right]\right) E_{ \pm}^{*}:
$$

где были использованы обозначения (3.20) и (3.22), (3.23). Снова в силу фермионного свойства операторов $\psi$ мы получаем, что в разложении экспоненты сохраняются только первьй и второй члены. Тогда в силу соотношений, доказанных в лемме 3.3 , мы перемешаем все зависяшие от $x$ операторы направо и, наконец, используя обозначение (3.21), получаем

$$
e^{ \pm i \varepsilon^{-1} V_{ \pm}^{\mp}(x)} e^{ \pm i \varepsilon^{-1} V_{ \pm}^{ \pm}(x)}=\frac{c \pm i x}{\varepsilon \sqrt{2 c}} R_{ \pm}^{*} \psi_{ \pm}(x),
$$

что при подстановке в (3.44) доказывает (3.27). 


\section{4. Свойства скалярного поля}

Как легко видеть, операторы $R_{+}$и $R_{-}$, введенные в $(3.21)$, взаимно антикоммутируют,

$$
\left\{R_{+}, R_{-}\right\}=0 \text {, }
$$

и в силу (2.16) и (3.41) они сдвигают операторы заряда:

$$
R_{ \pm}^{*} \Lambda_{ \pm} R_{ \pm}=\Lambda_{ \pm}-\varepsilon
$$

Коммутируюшие операторы, которые сохраняют все остальные соотношения данной алгебры, вводятся посредством преобразования

$$
\widehat{R}_{ \pm}=\exp \left( \pm \frac{i \pi}{2 \varepsilon} \Lambda_{\mp}\right) R_{ \pm}
$$

Тогда $\left[\widehat{R}_{+}, \widehat{R}_{-}\right]=0$ в силу $(4.2)$ и, поскольку операторы $\widehat{R}_{ \pm}$унитарны, сушествуют самосопряженные операторы $p_{ \pm}[41]$ такие, что

$$
\widehat{R}_{ \pm}=\exp \left(\frac{i p_{ \pm}}{2 \pi}\right)
$$

Эти операторы канонически сопряжены операторам заряда:

$$
\left[\Lambda_{ \pm}, p_{ \pm}\right]=i
$$

а потому они ограничены $[43]$.

Далее, по (3.43) и (4.2) имеем коммутационное соотношение

$$
\left[V_{ \pm}^{ \pm}(x) \mp i \Lambda_{ \pm} \log \frac{c \mp i x}{2 c}, R_{ \pm}\right]=0 .
$$

Введем операторы $d(k), k \in \mathbb{R}$, такие, что

$$
d(k)=\frac{1}{2 \pi i \varepsilon \sqrt{|k|}} \int d x e^{-i k x}\left[\theta(k) v_{+}(x)+\theta(-k) v_{-}(x)\right],
$$

так что по (2.21), (3.3), (3.16) и (2.16)

$$
V_{ \pm}^{ \pm}(x)= \pm \varepsilon \int_{0}^{\infty} \frac{d k}{\sqrt{k}}\left(e^{ \pm i k x}-e^{-k c}\right) d( \pm k) \pm i \Lambda_{ \pm} \log \frac{c \mp i x}{2 c} .
$$

Благодаря (3.18) и (3.19) и принимая во внимание, что операторы $V_{ \pm}$и $\Lambda_{ \pm}$коммутируют, получаем, что операторы $d(k)$ и $d^{*}(k)$ дают приводимые представления канонических бозонных операторов рождения-уничтожения:

$$
d(k) \Omega=0, \quad\left[d(k), d\left(k^{\prime}\right)\right]=\delta\left(k-k^{\prime}\right), \quad\left[d(k), \Lambda_{ \pm}\right]=0 .
$$

По (3.43) и (4.6) мы также получаем, что

$$
\left[R_{ \pm}, d(k)\right]=0
$$


а тогда

$$
\left[p_{ \pm}, d(k)\right]=0
$$

что доказьвает, что нулевая и осцилляторные моды разделены. Отметим также, что в соответствии с тем, что $V_{ \pm}^{ \pm}$и $V_{ \pm}^{\mp}$ хорошо определены как операторнозначные обобщенные функции, интегральньй член в правой части (4.8) возник автоматически регуляризованным в точке $k=0$.

Скалярное (вещественное, безмассовое, двумерное) поле вводится как формальньй логарифм произведения $\psi_{+} \psi_{-}$по (3.16):

$$
\phi(t, x)=\frac{1}{\sqrt{2}} \sum_{ \pm}\left\{ \pm V_{ \pm}^{\mp}(x \mp t) \pm V_{ \pm}^{ \pm}(x \mp t)+\varepsilon^{2} p_{ \pm}\right\}
$$

где временная эволюция (2.7) была принята во внимание. Это поле удовлетворяет каноническим коммутационным соотношениям:

$$
\left[\phi(t, x), \phi_{t}(t, y)\right]=i \delta(x-y)
$$

С другой стороны, в [41] было показано, что вакуумное среднее мономов этого поля по отношению к вакууму $\Omega$ не сушествует, поскольку векторы $p_{ \pm} \Omega$ не принадлежат областям определения операторов $\Lambda_{ \pm}$. Более того, скалярное поле $\phi$ не является вейлевым, поскольку равенство

$$
e^{i \alpha \Lambda_{ \pm}} e^{i \beta p_{ \pm}}=e^{-i \alpha \beta} e^{i \beta p_{ \pm}} e^{i \alpha \Lambda_{ \pm}}
$$

для вешественных констант $\alpha$ и $\beta$ вьполняяется лишш при $\beta \in \mathbb{Z}$.

Несмотря на все эти специфические свойства, $\phi(t, x)$ - хорошо определенная лоренщ-инвариантная операторнозначная обобшенная функция в $\mathscr{H}$ при произвольной положительной константе $c$. Поля, отвечающие различным значениям $c$, связаны посредством тривиального унитарного автоморфизма, но предел $c \rightarrow 0$ не существует. В рассмотренном здесь случае $x \in \mathbb{R}$ этот параметр обеспечивает необходимую инфракрасную регуляризащию всего построения и позволяет ввести операторы нулевых мод $\Lambda_{ \pm}, p_{ \pm}$. Таким образом (см. [41; ч. I] для подробностей), мы получаем следуюшие равенства для фермионного гильбертова пространства:

$$
\mathscr{H}=\bigoplus_{\lambda_{+}, \lambda_{-}=-\infty}^{+\infty} \mathscr{H}_{\lambda_{+}, \lambda_{-}}^{B}=\mathscr{H}^{d} \otimes \mathscr{H}_{+} \otimes \mathscr{H}_{-}
$$

Здесь, в первом равенстве, $\mathscr{H}_{\lambda_{+}, \lambda_{-}}^{B}$ суть бозонные пространства Фока, натянутые на векторы, полученные последовательньм применением оператора $d^{*}(k)$ к вакууму

$$
\Omega_{\lambda_{+}, \lambda_{-}}=R_{+}^{-\lambda_{+}} R_{-}^{-\lambda_{-}} \Omega, \quad \lambda_{ \pm}=0, \pm 1, \pm 2, \ldots
$$

В силу (4.10)

$$
d(k) \Omega_{\lambda_{+}, \lambda_{-}}=0,
$$

а по (4.2)

$$
\Lambda_{ \pm} \Omega_{\lambda_{+}, \lambda_{-}}=\varepsilon \lambda_{ \pm} \Omega_{\lambda_{+}, \lambda_{-}}
$$


Итак, в силу последнего равенства в (4.9) подпространства $\mathscr{H}_{\lambda_{+}, \lambda_{-}}^{B}$ являются собственными для операторов заряда $\Lambda_{ \pm}$, а потому они взаимно ортогональны. Во втором равенстве в (4.14) $\mathscr{H}^{d}$ - пространство Фока для операторов $d(k), d^{*}(k)$ и $\mathscr{H}_{ \pm}-$пространства, где действуют операторы $p_{ \pm}$и $\Lambda_{ \pm}$нулевых мод.

\section{5. Случай периодических полей}

$2 \pi$-периодическое поле $\widetilde{\psi}_{ \pm}$может быть построено через поле $\psi_{ \pm}$посредством конформной инвариантности, т.е. посредством стереографической проекции $e^{i \widetilde{x}}=$ $(c+i x) /(c-i x)$. Другими словами, мы вводим новую пространственную переменную каK

$$
\widetilde{x}=2 \arctan \frac{x}{c},
$$

где $c$ - некоторый вешественньй положительный параметр, которьй может быть выбран произвольно, но, как будет показано ниже, удобно выбрать его равным параметру $c$ инфракрасной регуляризации, которьй использовался вьше. При изменении $x$ монотонно от $-\infty$ до $+\infty$ переменная $\widetilde{x}$ пробегает от $-\pi$ до $+\pi$. На этом интервале мы вводим поля $\widetilde{\psi}_{ \pm}$посредством равенств

$$
\widetilde{\psi}_{ \pm}(\widetilde{x})=\frac{c-i x}{\sqrt{2 \varkappa}} \psi_{ \pm}(x)
$$

где $\psi_{ \pm}$определено в $(2.3)$, и продолжаем эти функции периодически:

$$
\widetilde{\psi}_{ \pm}(\widetilde{x}+2 \pi)=\widetilde{\psi}_{ \pm}(\widetilde{x}) .
$$

В силу (2.3) эти поля можно записать как

$$
\begin{aligned}
& \widetilde{\psi}_{+}(\widetilde{x})=\varepsilon\left\{\sum_{n=1}^{\infty} e^{-i n \widetilde{x}} a_{n}^{*}+\sum_{n=0}^{\infty} e^{+i n \widetilde{x}} b_{n}\right\}, \\
& \widetilde{\psi}_{-}(\widetilde{x})=\varepsilon\left\{\sum_{n=0}^{\infty} e^{i n \widetilde{x}} a_{-n}^{*}+\sum_{n=1}^{\infty} e^{-i n \widetilde{x}} b_{-n}\right\},
\end{aligned}
$$

где мы ввели операторы

$$
\begin{gathered}
a_{n}= \begin{cases}\int_{0}^{\infty} d k f_{n-1}(k) a(k), & n=1,2, \ldots, \\
\int_{0}^{\infty} d k f_{-n}(k) a(-k), & n=0,-1,-2, \ldots,\end{cases} \\
b_{n}= \begin{cases}\int_{0}^{\infty} d k f_{n}(k) b(k), & n=0,1,2, \ldots, \\
\int_{0}^{\infty} d k f_{-n-1}(k) b(-k), & n=-1,-2, \ldots,\end{cases}
\end{gathered}
$$

и где $f_{n}(k)$ означают функции, ассоциированные с полиномами Лагерра,

$$
f_{n}(k)=\frac{\sqrt{2 c}(-1)^{n}}{n !} e^{k c} \frac{\partial^{n}}{\partial k^{n}}\left(k^{n} e^{-2 k c}\right), \quad n=0,1,2, \ldots, \quad k \geqslant 0 .
$$


По (2.2) операторы $a_{n}$ и $b_{n}, n=0, \pm 1, \pm 2, \ldots$, являются операторами уничтожения в пространстве Фока $\mathscr{H}$ с тем же самьм вакуумом $\Omega \in \mathscr{H}$,

$$
a_{n} \Omega=b_{n} \Omega=0, \quad n=0, \pm 1, \pm 2, \ldots,
$$

а принимая во внимание известные свойства ортогональности и полноты функций $f_{n}$, мы получаем, что эрмитово сопряженные операторы $a_{n}^{*}, b_{n}^{*}$ являются операторами рождения и они растягивают то же самое пространство $\mathscr{H}$ и что вьполнены канонические и антикоммутационные соотношения

$$
\left[a_{m}, a_{n}^{*}\right]_{+}=\delta_{m n},\left[b_{m}, b_{n}^{*}\right]_{+}=\delta_{m n}, \quad m, n=0, \pm 1, \pm 2, \ldots,
$$

с другими антикоммутаторами, равньми нулю. Тогда также

$$
\left[\widetilde{\psi}_{ \pm}(\widetilde{x}), \widetilde{\psi}_{ \pm}^{*}(\widetilde{y})\right]_{+}=\hbar \widetilde{\delta}(\widetilde{x}-\widetilde{y})
$$

где $\widetilde{\delta}-2 \pi$-периодическая $\delta$-функция. Благодаря (5.1) поля $\widetilde{\psi}_{ \pm}$обладают следуюшими вакуумньми ожиданиями:

$$
\left(\widetilde{\Omega}, \widetilde{\psi}_{ \pm}(\widetilde{x}) \widetilde{\psi}_{ \pm}^{*}(\widetilde{y}) \widetilde{\Omega}\right)= \pm \varepsilon^{2}\left(1-e^{i(\widetilde{x}-\widetilde{y}) \mp 0}\right)^{-1}=\left(\widetilde{\Omega}, \widetilde{\psi}_{ \pm}^{*}(\widetilde{x}) \widetilde{\psi} \pm(\widetilde{y}) \widetilde{\Omega}\right) e^{i(\widetilde{x}-\widetilde{y})}
$$

что вытекает из (2.6).

Токи введенных полей определяются (ср. (2.11)) как

$$
\widetilde{v}_{ \pm}(\widetilde{x})=\varepsilon^{-1}: \widetilde{\psi}_{ \pm}^{*} \widetilde{\psi}_{ \pm}:(\widetilde{x})
$$

так что по (5.2)

$$
\widetilde{v}_{ \pm}(\widetilde{x})=\frac{c^{2}+x^{2}}{2 c} v_{ \pm}(x)
$$

Токи удовлетворяют коммутационному соотношению

$$
\left[\widetilde{v}_{ \pm}(\widetilde{x}), \widetilde{v}_{ \pm}(\widetilde{y})\right]=i \hbar \widetilde{\delta}^{\prime}(\widetilde{x}-\widetilde{y}),
$$

которое совместно с (2.15) в силу $(5.1),(5.14)$ и равенства

$$
\widetilde{\delta}^{\prime}(\widetilde{x}-\widetilde{y})=(2 c)^{-2}\left(c^{2}+x^{2}\right)\left(c^{2}+y^{2}\right) \delta^{\prime}(x-y) .
$$

Операторы зарядов вводятся как

$$
\widetilde{\Lambda}_{ \pm}=\frac{1}{2 \pi} \int_{0}^{2 \pi} d \widetilde{x} \widetilde{v}_{ \pm}(\widetilde{x}),
$$

так что по (5.14) и (2.16) они совпадают с этими же операторами для случая бесконечной прямой.

Разложение (2.20), (2.21) под действием (5.1) сводится к разложению на слагаемые, допускаюшие аналитическое продолжение по переменной $\widetilde{x}$ внутрь и наружу единичной окружности. Тогда по (3.3), (3.4), (3.16), (3.17) и (5.14) мы получаем для первообразных токов равенство

$$
\widetilde{V}_{ \pm}^{ \pm}(\widetilde{x})=V_{ \pm}^{ \pm}(x)
$$


а в силу (4.8)

$$
\widetilde{V}_{ \pm}^{ \pm}(\widetilde{x})= \pm \varepsilon \sum_{n=1}^{\infty} \frac{e^{ \pm i n \widetilde{x}}}{\sqrt{n}} d_{ \pm n}-\frac{\widetilde{x}}{4 \pi} \Lambda_{ \pm}
$$

где волна над $\Lambda_{ \pm}$была опушена ввиду (5.16). Здесь мы ввели операторы $d_{n}$ как

$$
\sqrt{|n|} d_{ \pm n}=\frac{1}{\sqrt{2 c}} \int_{0}^{\infty} d k\left[\left(c+\frac{\partial}{\partial k}\right) f_{ \pm n}(k)\right] \sqrt{|k|} d( \pm k), \quad n= \pm 1, \pm 2, \ldots
$$

где операторы $d(k)$ были определены в (4.7). Операторы $d_{n}$ - бозонные операторы, они удовлетворяют равенствам

$$
d_{n} \Omega=0, \quad\left[d_{m}, d_{n}^{*}\right]=\delta_{m, n}, \quad m, n= \pm 1, \pm 2, \ldots,
$$

и коммутируют с операторами заряда и сплетаюшими операторами. Мы видим, что благодаря выбору постоянной $c$ член нулевой моды выделен из суммы в (5.18), a paвенство (5.19) превращается в тождество при $n=0$.

Суммируя, соответствие Колемана-Мандельштама для $\widetilde{\psi}_{ \pm}(\widetilde{x})$ записьваем в форме (3.27):

$$
\widetilde{\psi}_{ \pm}(\widetilde{x})=\frac{1}{\sqrt{2 \pi}} e^{ \pm i \varepsilon^{-1} \widetilde{V}_{ \pm}^{\mp}(\widetilde{x})} R_{ \pm} e^{ \pm i \varepsilon^{-1} \widetilde{V}_{ \pm}^{ \pm}(\widetilde{x})} .
$$

В случае, когда рассматривается только одна из киральностей (“+” или “_”), (5.21) является реализацией в терминах фермионных операторов результата, данного в [23]. В (5.21) $R_{ \pm}$- это те же самые сплетающие операторы, которые были введены в $(3.21)$, где теперь в силу $(2.3),(2.21),(3.22),(3.23)$ и (5.6), (5.7) мы можем записать, что

$$
\begin{array}{ll}
a_{+}=a_{1}, & a_{-}=a_{0}, \\
b_{+}=b_{0}, & b_{-}=b_{-1},
\end{array}
$$

и для краткости мы опускаем выражения для операторов $E_{ \pm}$через посредство операторов $a_{n}$ и $b_{n}$, которые могут быть легко вьведены.

В силу (5.18) скалярное поле задается аналогом с волной равенства (4.12) и имеет свойства, аналогичные случаю бесконечной оси.

\section{6. Иерархия интегрируемых и явно решаемых моделей}

Бозон-фермионное соответствие в том виде, как оно было представлено в предыдуших разделах, должно быть применимо к квантованию релятивистских интегрируемых моделей. Действительно, впервые оно возникло при квановании модели sine-Гopдон [2]-[5]. Благодаря ему квантовьй гамильтониан этой модели был сведен к гамильтониану массивной модели Тирринга, полная интегрируемость которой в классическом случае также известна. Эта редукция была вьполнена в терминах безмассовых фермионных полей, поэтому получившийся гамильтониан был плохо определен. Принимая во внимание, что аналог бозон-фермионного соответствия для массивного случая все еще неизвестен, естественно полагать, что область приложений данного соответствия дается “безмассовыми” интегрируемьми моделями, типа уравнения Лиувилля. С другой стороны, квантование моделей, определяемых скобкой Гарднера-Захарова-Фаддеева (1.4), достаточно непосредственно ввиду коммутационного 
равенства (2.15). Такой подход к квантованию уравнения КдФ был развит в [4], [5], см. также раздел 8 ниже. В [4] и [5], в частности, было показано, что в случае бездисперсного КдФ соответствующая эволюция фермионного поля линейна. Соответственно, в этом разделе мы исследуем проблему описания всех нелинейных взаимодействий бозонного поля, удовлетворяющего (2.15), которые при фермионизации, задаваемой равенством (2.11), приводят к линейньм эволюционньм уравнениям для фермионного поля $\psi$. Такой подход затрагивает лишь фермионное поле одной киральности, скажем -, так что мы упростим ниже обозначения, положив

$$
\psi(x)=\psi_{-}(x), \quad v(x)=v_{-}(x), \quad \text { и т.д. }
$$

Любая эволюция, линейная по отношению к $\psi$, задается гамильтонианом, которьй включает в себя лишь комбинации типа $\psi^{*} \psi, \psi \psi$ и $\psi^{*} \psi^{*}$. Но в силу (3.27) только первая из них может быть записана через посредство тока $v$ : обе другие включают в себя сплетаюшие операторы $R$, см. (3.39). Итак, нас интересуют только гамильтонианы, порождаемые произведением : $\psi^{*}(x) \psi(y)$ :. Выражение этого произведения через бозонное поле (ток) $v(x)$ непосредственно следует из (3.27), равно как и факт, что оно не включает в себя ни сплетаюшего оператора $R$, ни параметра инфракрасной регуляризации $c$. Таким образом, выражение для этой билинейной комбинации проше, чем выражение для самих фермионных полей, и по этой причине мы даем здесь независимый вывод этого соотношения непосредственно в терминах тока $v(x)$, см. [44]. Аналог такого соотношения в смысле формальных рядов известен в литературе по симметриям иерархий КП и КдФ (см. [8]).

Теорема 6.1. Для любых вещественных $x$ и у тождество

$$
\varepsilon^{-1}: \psi^{*}(x+y) \psi(x-y):=\varepsilon \frac{\vdots \exp \left(i \varepsilon^{-1} \int_{x-y}^{x+y} d x^{\prime} v\left(x^{\prime}\right)\right) \vdots-1}{2 i y},
$$

әде в определено в (2.4), выполняется в смысле операторнозначных обобщенных функиий пространственной переменной $x$, гладко зависящих от $y$.

ДокАЗАТЕльСтво. Благодаря (2.34) и (2.36) (для нижнего знака) мы имеем

$$
\begin{aligned}
-i \varepsilon \frac{\partial}{\partial y} & \psi^{*}(x+y) \psi(x-y) \\
= & v^{+}(x+y) \psi^{*}(x+y) \psi(x-y)+\psi^{*}(x+y) \psi(x-y) v^{-}(x-y) \\
& +\psi^{*}(x+y)\left(v^{-}(x+y)+v^{+}(x-y)\right) \psi(x-y) \\
\equiv & \left(v^{+}(x+y)+v^{+}(x-y)\right) \psi^{*}(x+y) \psi(x-y) \\
& +\psi^{*}(x+y) \psi(x-y)\left(v^{-}(x+y)+v^{-}(x-y)\right) \\
& +\left[\psi^{*}(x+y), v^{+}(x-y)\right] \psi(x-y)+\psi^{*}(x+y)\left[v^{-}(x+y), \psi(x-y)\right] .
\end{aligned}
$$

Принимая во внимание (2.14) и (2.21), мы можем записать это равенство в виде

$$
\begin{aligned}
-i \varepsilon & \frac{\partial}{\partial y} \psi^{*}(x+y) \psi(x-y) \\
= & \left(v^{+}(x+y)+v^{+}(x-y)+\frac{i \varepsilon}{2(y-i 0)}\right) \psi^{*}(x+y) \psi(x-y) \\
& \quad+\psi^{*}(x+y) \psi(x-y)\left(v^{-}(x+y)+v^{-}(x-y)+\frac{i \varepsilon}{2(y-i 0)}\right) .
\end{aligned}
$$


Пусть

$$
F(x, y)=\varepsilon^{-1}: \psi^{*}(x+y) \psi(x-y):,
$$

т.е. $F$ обозначает левую часть (6.2). Тогда по определению фермионного виковского упорядочения мы имеем $\psi^{*}(x+y) \psi(x-y)=\varepsilon F(x, y)-i \varepsilon^{2}(2 y-i 0)^{-1}$. Заменим $\psi^{*} \psi$ в (6.3) посредством этого равенства и учтем, что степени обобщенной функции $(y-i 0)^{-1}$ хорошо определены. Тогда мы получаем следуюшее дифференциальное уравнение для $F(x, y)$ :

$$
\frac{\partial F(x, y)}{\partial y}=\frac{i}{\varepsilon}:(v(x+y)+v(x-y)) F(x, y) \vdots+\frac{v(x+y)+v(x-y)-2 F(x, y)}{2(y-i 0)} .
$$

В то же время по (2.11) и (6.4) при $y=0$

$$
F(x, 0)=v(x),
$$

так что член $i 0$ в знаменателе может быть опушен и мы получаем

$$
\frac{\partial F(x, y)}{\partial y}=\frac{i}{\varepsilon}:(v(x+y)+v(x-y)) F(x, y) \vdots+\frac{v(x+y)+v(x-y)-2 F(x, y)}{2 y} .
$$

Легко проверить непосредственно, что правая часть (6.2) удовлетворяет тому же дифференциальному уравнению по $y$ и тому же граничному условию (6.5), так что

$$
F(x, y)=\varepsilon \frac{\vdots \exp \left(i \varepsilon^{-1} \int_{x-y}^{x+y} d x^{\prime} v\left(x^{\prime}\right)\right) \vdots-1}{2 i y},
$$

что доказьвает (6.2). В силу (6.4) очевидно, что $F(x, y)$, действительно, есть операторнозначная обобшенная функция по переменной $x$, которая является гладкой, бесконечно дифференцируемой функцией $y$.

Введем

$$
\left.F_{n}(x) \equiv\left(\frac{\varepsilon \partial_{y}}{2 i}\right)^{n} F(x, y)\right|_{y=0}=\frac{\varepsilon^{n-1}}{(2 i)^{n}} D^{n}\left(: \psi^{*} \cdot \psi:\right)(x),
$$

где во втором равенстве мы использовали обозначение для производной Хироты [8], которая в обшем случае двух функций $f(x)$ и $g(x)$ определяется как

$$
D^{n}(f \cdot g)(x)=\lim _{y \rightarrow 0} \frac{\partial^{n}}{\partial y^{n}} f(x+y) g(x-y), \quad n=1,2, \ldots
$$

В частности, по (6.5) мы получаем, что

$$
\begin{aligned}
& F_{0}(x)=v(x), \\
& F_{1}(x)=\frac{1}{2 i} D\left(: \psi^{*} \cdot \psi:\right)(x)
\end{aligned}
$$

(ср. (2.31)), которые, соответственно, являются плотностями тока и энергии-импульса безмассового ферми-поля. Итак, уравнение (6.2) дает связь производных Хироты фермионных полей с полиномами тока и его производных. Все $F_{n}(x)$ в силу (6.8) являются 
самосопряженньми операторнозначными обобщенными функциями в фоковском пространстве $\mathscr{H}$ и по (6.7) мы получаем рекурсионные соотношения

$$
F_{2 n+1}(x)=\frac{1}{2 n+2} \sum_{m=0}^{n} \frac{(-i \varepsilon / 2)^{2 m}(2 n+1) !}{(2 m) !(2(n-m)) !} v^{(2 m)}(x) F_{2(n-m)}(x) \vdots, \quad n=0,1,2, \ldots,
$$

и

$$
\begin{aligned}
F_{2 n}(x)= & \frac{1}{2 n+1} \sum_{m=0}^{n-1} \frac{(-i \varepsilon / 2)^{2 m}(2 n) !}{(2 m) !(2(n-m)-1) !} \vdots v^{(2 m)}(x) F_{2(n-m)-1}(x) \\
& +\left(\frac{\varepsilon}{2 i}\right)^{n} \frac{v^{(2 n)}(x)}{2 n+1}, \quad n=1,2, \ldots,
\end{aligned}
$$

где $F_{0}$ дано в (6.10). Приведем простейшие низшие примеры операторов $F_{n}(x)$ :

$$
\begin{aligned}
& F_{1}(x)=\frac{1}{2}: v^{2} \vdots(x), \\
& F_{2}(x)=\frac{1}{3} \vdots v^{3} \vdots(x)-\frac{\varepsilon^{2} v_{x x}(x)}{12}, \\
& F_{3}(x)=\frac{1}{4} \vdots v^{4} \vdots(x)-\frac{\varepsilon^{2}}{4} \vdots(x) v_{x x}(x) \vdots \\
& F_{4}(x)=\frac{1}{5} \vdots v^{5} \vdots(x)-\frac{\varepsilon^{2}}{2} \vdots v^{2}(x) v_{x x}(x) \vdots+\frac{\varepsilon^{4} v_{x x x x}(x)}{80} .
\end{aligned}
$$

По определению (6.4) оператор $F(x, y)$ удовлетворяет коммутационному соотношению

$$
\begin{aligned}
{\left[F(x, y), F\left(x^{\prime}, y^{\prime}\right)\right]=} & -\varepsilon^{-1} \delta\left(x-x^{\prime}+y+y^{\prime}\right) F\left(x+y^{\prime}, y+y^{\prime}\right) \\
& +\varepsilon^{-1} \delta\left(x-x^{\prime}-y-y^{\prime}\right) F\left(x^{\prime}+y, y+y^{\prime}\right) \\
& +i \frac{\delta\left(x-x^{\prime}+y+y^{\prime}\right)-\delta\left(x-x^{\prime}-y-y^{\prime}\right)}{y+y^{\prime}}
\end{aligned}
$$

которое порождает соответствующие коммутационные соотношения для $F_{m}$ (близко связанные с представлением алгебры $\left.\mathfrak{g l}_{\infty}\right)$. Только младшие члены, $F_{0}$ и $F_{1}$, образуют замкнутые подалгебры:

$$
\begin{aligned}
& {\left[F_{0}(x), F_{0}\left(x^{\prime}\right)\right]=i \delta^{\prime}\left(x-x^{\prime}\right)} \\
& {\left[F_{0}(x), F_{1}\left(x^{\prime}\right)\right]=i \delta^{\prime}\left(x-x^{\prime}\right) F_{0}\left(x^{\prime}\right)} \\
& {\left[F_{1}(x), F_{1}\left(x^{\prime}\right)\right]=i\left\{F_{1}(x)+F_{1}\left(x^{\prime}\right)\right\} \delta^{\prime}\left(x-x^{\prime}\right)-\frac{i \varepsilon^{2}}{12} \delta^{\prime \prime \prime}\left(x-x^{\prime}\right) .}
\end{aligned}
$$

Оператор $F(x, y)$ допускает интегрирование по $x$ вдоль всей оси, и результат интегрирования - хорошо определенный оператор в фермионном фоковском пространстве $\mathscr{H}$. Действительно, по (2.3) и (6.4)

$$
\int d x F(x, y)=\frac{-1}{\varepsilon} \int_{-\infty}^{0} d k\left(e^{2 i k y} a^{*}(k) a(k)-e^{-2 i k y} b^{*}(k) b(k)\right),
$$


где выражение в правой части нормально упорядочено и имеет вид рождение $\times$ уничтожение, так что благодаря (2.2)

$$
\int d x F(x, y) \Omega=0
$$

для любого $y$. Отсюда мы вьводим, что все операторы

$$
H_{n} \equiv \int d x F_{n}(x)=\frac{-1}{\varepsilon} \int_{-\infty}^{0} d k(\varepsilon k)^{n}\left(a^{*}(k) a(k)-(-1)^{n} b^{*}(k) b(k)\right)
$$

хорошо определены, самосопряжены и обнуляют вакуум $\Omega$. Для нечетных $n$ они положительно определены. В то же время по (6.18) мы получаем

$$
\left[\int d x F(x, y), \int d x^{\prime} F\left(x^{\prime}, y^{\prime}\right)\right]=0
$$

для любых $y$ и $y^{\prime}$. Это, в частности, означает, что

$$
\left[H_{m}, H_{n}\right]=0, \quad m, n=0,1,2, \ldots
$$

Другими словами, эти операторы определяют коммутируюшие потоки на пространстве $\mathscr{H}$, и мы можем ввести иерархию интегрируемых временных эволюций, порождаемых этими потоками, как

$$
v_{t_{m}}(x)=i\left[H_{m}, v(x)\right], \quad m=0,1,2, \ldots,
$$

так что по (6.26): $\left(\partial_{t_{m}} \partial_{t_{n}}-\partial_{t_{n}} \partial_{t_{m}}\right) v(x)=0$ для любых $m$ и $n$ (мы не указьваем временную зависимость в тех случаях, когда это не необходимо). С другой стороны, по (6.18)

$$
\begin{aligned}
& {\left[\int d x F(x, y), v\left(x^{\prime}\right)\right]=\varepsilon^{-1}\left[F\left(x^{\prime}+y, y\right)-F\left(x^{\prime}-y, y\right)\right]} \\
& \quad \equiv \frac{1}{2 i y}\left\{\vdots \exp \left(i \varepsilon^{-1} \int_{x^{\prime}}^{x^{\prime}+2 y} d \xi v(\xi)\right) \vdots-\vdots \exp \left(i \varepsilon^{-1} \int_{x^{\prime}-2 y}^{x^{\prime}} d \xi v(\xi)\right) \vdots\right\},
\end{aligned}
$$

что приводит к весьма нелинейным (полиномиальньм) динамическим уравнениям для $v(x)$ во всех случаях за исключением $t_{0}$ и $t_{1}$. В силу $(6.8),(6.24)$ и $(6.27)$ мы имеем:

$$
\begin{aligned}
& v_{t_{0}}(x)=0, \\
& v_{t_{1}}(x)=v_{x}(x),
\end{aligned}
$$

а в ситуации общего положения

$$
v_{t_{n}}(x)=\frac{\partial}{\partial x} \sum_{m=0}^{\left[\frac{n-1}{2}\right]} \frac{(i \varepsilon / 2)^{2 m} n ! \partial_{x}^{2 m} F_{n-2 m-1}(x)}{(n-2 m-1) !(2 m+1) !}, \quad n=1,2, \ldots
$$


Простейшие примеры следуюшие:

$$
\begin{aligned}
& v_{t_{2}}(x)=\partial_{x} \vdots v^{2}:(x), \\
& v_{t_{3}}(x)=\partial_{x}\left(\vdots v^{3}:(x)-\frac{\varepsilon^{2}}{2} v_{x x}(x)\right), \\
& v_{t_{4}}(x)=\partial_{x}\left(\vdots v^{4}:(x)-2 \varepsilon^{2}: v v_{x x} \vdots(x)-\varepsilon^{2}: v_{x}^{2} \vdots(x)\right) .
\end{aligned}
$$

Эти полиномиальные взаимодействия непосредственно связаны с иерархией Кд $\Phi$ : вторая эволюция есть не что иное, как бездисперсное квантовое уравнение Кд $\Phi$ (ср. [6]), третья эволюция совпадает с модифицированным уравнением Кд $\Phi$ для некоторого конкретного значения константы связи, и т. д. В следующих разделах мы обсудим случаи уравнений КдФ и мКдФ более подробно. Здесь же подчеркнем, что, несмотря на сильно нелинейную форму всех этих уравнений в терминах поля $v$, все они дают линейные эволюции для фермионов, как и должно быть по построению. Вводя временную зависимость $\psi(x)$ как $\psi_{t_{m}}=i\left[H_{m}, \psi\right]$, мы получаем по $(6.24)$

$$
\psi_{t_{m}}(x)=\frac{1}{i \varepsilon}\left(i \varepsilon \partial_{x}\right)^{m} \psi(x),
$$

или по $(2.3) \psi_{t_{m}}(k)=(i \varepsilon)^{-1}(-\varepsilon k)^{m} \psi(k)$. Пусть теперь $\psi\left(t_{m}, x\right), v\left(t_{m}, x\right)$ и $F\left(t_{m}, x, y\right)$ суть операторы с временной эволюцией, заданной некоторым $H_{m}$, и определяемые условием, что при $t_{m}=0$ они равны $\psi(x), v(x)$ и $F(x, y)$ соответственно. В силу $(6.23)$ определения обоих нормальных произведений не зависят от времени. Это означает, что введенные операторы связаны в произвольньй момент $t_{m}$ посредством тех же самых уравнений $(2.11),(6.4),(6.7)$ и $(6.10)$, что и при $t_{m}=0$. В частности, по $(6.4)$

$$
F\left(t_{m}, x, y\right)=\frac{1}{\varepsilon}: \psi^{*}\left(t_{m}, x+y\right) \psi\left(t_{m}, x-y\right): .
$$

Тогда благодаря (2.3), (6.4) и (6.35) мы получаем явное выражение для решения $F\left(t_{m}, x, y\right)$ через посредство его начального значения $F(x, y)$ :

$$
\begin{aligned}
F\left(t_{m}, x, y\right)= & \frac{2}{(2 \pi)^{2}} \int d x^{\prime} \int d y^{\prime} \int d k \int d p F\left(x-x^{\prime}, y-y^{\prime}\right) \\
& \times \exp \left(i(k-p) x^{\prime}+i(k+p) y^{\prime}+i \varepsilon^{m-1}\left(k^{m}-p^{m}\right) t_{m}\right) .
\end{aligned}
$$

В силу (6.8) и (6.10) мы получаем для $y=0$ :

$$
\begin{aligned}
v\left(t_{m}, x\right)= & \frac{2}{(2 \pi)^{2}} \int d x^{\prime} \int d y^{\prime} \int d k \int d p F\left(x-x^{\prime}, y^{\prime}\right) \\
& \times \exp \left(i(k-p) x^{\prime}-i(k+p) y^{\prime}+i \varepsilon^{m-1}\left(k^{m}-p^{m}\right) t_{m}\right) .
\end{aligned}
$$

Подставляя здесь $F(x, y)$ из (6.7), получаем решение $m$-го уравнения иерархии $(6.27)$, выраженное через его начальные данные $v(x)$ :

$$
\begin{aligned}
v\left(t_{m}, x\right)= & \frac{1}{(2 \pi)^{2}} \int d x^{\prime} \int d y^{\prime} \int d k \int d p \frac{\vdots \exp \left(i \varepsilon^{-1} \int_{x-x^{\prime}-\varepsilon y^{\prime}}^{x-x^{\prime}+\varepsilon y^{\prime}} d x^{\prime \prime} v\left(x^{\prime \prime}\right)\right) \vdots-1}{2 i y^{\prime}} \\
& \times \exp \left(i k x^{\prime}-i p y^{\prime}+i \frac{t_{m}}{2^{m} \varepsilon}\left[(p+\varepsilon k)^{m}-(p-\varepsilon k)^{m}\right]\right)
\end{aligned}
$$


Обобщение на случай, когда временная эволюция задается линейной комбинацией гамильтонианов $H_{m}$, очевидно.

Итак, мы видим, что все эти модели не только вполне интегрируемы, но и явно решаемы в фермионном фоковском пространстве $\mathscr{H}$. С другой стороны, для оператора заряда (2.16) мы имеем по (6.24) $\Lambda=H_{0}$, т.е. он коммутирует со всеми операторами, участвуюшими в (6.39). Тогда это равенство можно редуцировать на нулевой (или любой другой, фиксированный) зарядовый сектор $\mathscr{H}$, которьй есть в точности стандартное бозонное фоковское пространство в силу (4.14). Таким образом, (6.39) дает явное решение иерархии (6.27) в бозонном фоковском пространстве.

\section{7. Классический предел иерархии (6.31)}

Коэффициенты бозонных уравнений движения (6.30)-(6.34) однозначно (с точностью до обшего множителя) фиксируются рекурсионными соотношениями (6.12), (6.13). Действительно,

$$
v(x) \rightarrow a v(a x)
$$

- единственное каноническое масштабное преобразование, унитарно порождаемое в $\mathscr{H}$. Здесь постоянная $a>0$, так что определение (2.21) положительной и отрицательной частей $v$ сохраняется. Это преобразование порождает преобразования:

$$
\psi(x) \rightarrow \sqrt{a} \psi(a x), \quad F(x, y) \rightarrow a F(a x, a y), \quad F_{n}(x) \rightarrow a^{n} F_{n}(a x),
$$

которые совместны с (6.10)-(6.13). Тогда по (6.24) $H_{n} \rightarrow a^{n-1} H_{n}$ и благодаря (6.31) преобразование (7.1) может быть скомпенсировано перенормировкой времен: $t_{n} \rightarrow$ $a^{1-n} t_{n}$.

Потоки, заданные в (6.10)-(6.17), близки к потокам иерархии КдФ [29]: они полиномиальны по $v(x)$ и его производным и имеют те же самые старшие члены. В то же время, как мы уже указьвали вьше, низший нетривиальньй пример (6.15) являет-

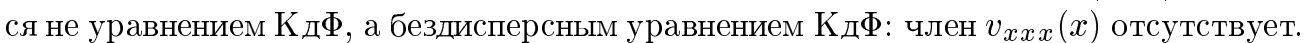
В следуюшем уравнении (6.16) некоторые члены второго уравнения иерархии КдФ также пропущены и, как мы показьваем в разделе 9, оно является модифищированным уравнением Кд $\Phi$ с некоторьм специальным значением константы взаимодействия. Как следует из предыдущего, общее значение этой константы не может быть получено за счет преобразования (7.2). Уравнения (6.16), (6.17), равно как и все высшие уравнения, уже включают в себя члены с производными, так что эти уравнения уже не являются, строго говоря, бездисперсными. С другой стороны, коэффициенты всех таких членов всех коммутируюших потоков, введенных в разделе 6 , пропорциональны степеням $\varepsilon^{2}$, т.е. $\hbar$, см. (2.4). В силу (6.10) и (6.12), (6.13) легко видеть, что в пределе $\hbar \rightarrow 0$

$$
F_{m}(x) \rightarrow \frac{v^{m+1}(x)}{m+1}
$$

так что по (6.31) мы получаем классические предельные уравнения

$$
\partial_{t_{m}} v\left(t_{m}, x\right)=m v^{m-1}\left(t_{m}, x\right) v_{x}\left(t_{m}, x\right),
$$

т.е. бездисперсную иерархию Кд $\Phi$. Решение начальной задачи для $m$-го уравнения может быть записано [29] в параметрической форме как

$$
x=s-m t_{m} v^{m-1}(s), \quad v\left(t_{m}, x\right)=v(s),
$$


где $v(x)$ - начальные данные, а $s$ определяется первым равенством. Это решение, как известно, описьвает переворот фронта, так что начальная задача для уравнений (7.4) не имеет глобального решения. Вопреки этому уравнение (6.39) дает глобальное решение квантовой иерархии (6.31). Легко видеть, что в пределе $\varepsilon \rightarrow 0$ (т.е. $\hbar \rightarrow 0$ ) мы получаем из (6.39)

$$
v\left(t_{m}, x\right)=\frac{1}{(2 \pi)^{2} i} \int d x^{\prime} \int d y^{\prime} \int d k \int d p \frac{e^{i y^{\prime} v\left(x-x^{\prime}\right)}-1}{y^{\prime}} e^{i k x^{\prime}-i p y^{\prime}+i m t_{m} k p^{m-1}}
$$

так что для классического предела равенства (6.8) мы получаем представление

$$
v\left(t_{m}, x\right)=\int d p\left[\theta\left(v\left(x+m t_{m} p^{m-1}\right)-p\right)-\theta(-p)\right]
$$

которое до переворота фронта совпадает с (7.5) (здесь $\theta(p)$ означает ступенчатую функцию). Суммируя, естественно назьвать иерархию, введенную в разделе 6, квантовой бездисперсной иерархией КдФ.

\section{8. Квантование уравнения $K_{\text {д } \Phi}$}

В случае общей (не бездисперсной) интегрируемой модели, конечно, явную интегрируемость ожидать не приходится. Тем не менее, изложенная выше техника полезна при рассмотрении квантовых версий таких моделей. В этом и последующем разделах мы продемонстрируем ее применимость к уравнениям КдФ и мКдФ. Снова фермионизация скобки (1.4) дается (2.11) и (2.15), и мы снова опускаем индекс киральности, см. (6.1). Квантовьй гамильтониан - упорядоченное по бозонам выражение (1.7),

$$
H_{\mathrm{KdV}}=\int d x: v^{3}(x)+\frac{1}{2} v_{x}^{2}(x)
$$

так что по (6.15) и $(6.17)$

$$
H_{\mathrm{KdV}}=3 H_{2}+\frac{1}{2} \int d x \vdots v_{x}^{2}:(x)
$$

Итак, оба слагаемых этого гамильтониана являются хорошо определенными операторами, и он не требует никакой регуляризации. Наиболее сингулярная часть гамильтониана (8.1), которая была третьего порядка по бозонным операторам, теперь имеет только второй порядок по фермионньм: по (6.8) и (6.24)

$$
H_{2}=\int d x: \psi_{x}^{*} \psi_{x}:(x)
$$

В то же время по $(2.20),(2.21)$ и $(2.26)$

$$
\int d x: v_{x}^{2}:(x)=2 \int d x v_{x}^{+}(x) v_{x}^{-}(x)
$$


так что слагаемые в (8.2) билинейны либо по фермионным, либо по бозонньм операторам рождения-уничтожения и имеют диагональную форму, т.е. они содержат только члены типа "рождение ×уничтожение". Этот гамильтониан зануляет вакуум,

$$
H_{\mathrm{KdV}} \Omega=0
$$

но первое слагаемое в (8.1) не является положительно определенным, как следует из (6.24). С другой стороны, равенство (8.5) показывает, что вакуумные ожидания одновременных произведений полей не зависят от времени и поэтому оба типа виковского произведения совместны с временной эволюцией. Эволюция, заданная гамильтонианом (8.1),

$$
v_{t} \equiv i\left[H_{\mathrm{KdV}}, v\right]=6 \vdots v v_{x} \vdots-v_{x x x}
$$

есть в точности квантовая версия уравнения (1.1), нормально упорядоченная по бозонным операторам. В силу (2.11) и равенства $\vdots v^{2}:=-i D\left(: \psi^{*} \cdot \psi:\right)$, которое следует из (6.11) и (6.14), можно переписать (8.6) в виде:

$$
\frac{\partial}{\partial t}: \psi^{*} \psi:(x)=\frac{\partial}{\partial x}\left(-3 i \varepsilon D\left(: \psi^{*} \cdot \psi:\right)(x)-\frac{\partial^{2}}{\partial x^{2}}: \psi^{*} \psi:(x)\right),
$$

билинейном по отношению к фермионным полям. Равенство (8.7) можно рассматривать как "квантовую форму Хироты” уравнения КдФ. С другой стороны, временная эволюция $\psi_{t} \equiv i\left[H_{\mathrm{KdV}}, \psi\right]$ фермионного поля нелинейна:

$$
\begin{aligned}
\psi_{t}(x) & =3 i \varepsilon \psi_{x x}(x)+2 \pi i \varepsilon \dot{v}_{x x} \psi:(x) \\
& \equiv 3 i \varepsilon \psi_{x x}(x)-\frac{2 \pi \varepsilon^{2}}{3} \psi_{x x x}(x)+2 \pi i \varepsilon: v_{x x} \psi:(x),
\end{aligned}
$$

где первая строка вытекает из (6.35) и (2.14), а вторая из (2.36) при $n=2$.

Исследование спектра квантового гамильтониана заслуживает отдельного рассмотрения. Здесь мы покажем лишш, что в фермионном фоковском пространстве сушествует состояние, которое может быть названо односолитонным в том смысле, что среднее поля $v$ по этому состоянию равно классическому односолитонному решению

$$
v_{1-\text { solit }}=-\frac{2 \varkappa^{2}}{\cosh ^{2} \varkappa\left(x-4 \varkappa^{2} t-x_{0}\right)},
$$

по крайней мере при нулевом (или любом фиксированном) моменте времени. Для этого рассмотрим унитарное преобразование

$$
\widehat{\psi}(x)=W \psi(x) W^{*}, \quad \widehat{v}(x)=W v(x) W^{*}
$$

такое, что

$$
\widehat{\psi}(x)=e^{i \gamma(x)} \psi(x),
$$

где $\gamma(x)$ - гладкая, вешественная $c$-значная функщия. Тогда по (2.26) и (2.11) мы получаем для тока соотношение

$$
\widehat{v}(x)=v(x)-\varepsilon \gamma^{\prime}(x),
$$


так что для существования оператора $W$ в $\mathscr{H}$ необходимо наложить асимптотическое условие

$$
\lim _{x \rightarrow+\infty} \gamma(x)-\lim _{x \rightarrow-\infty} \gamma(x)=2 \pi n, \quad n \in \mathbb{Z},
$$

на эту функцию. По (8.10) и (8.12) $\left(W^{*} \Omega, v(x) W^{*} \Omega\right)=-\varepsilon \gamma^{\prime}(x)$, так что для того чтобы правая часть равнялась солитону (8.9) при $t=0$, мы полагаем

$$
\gamma(x)=\frac{2 \varkappa}{\varepsilon} \tanh \varkappa\left(x-x_{0}\right) .
$$

Условие (8.13) тогда влечет за собой квантование солитонной переменной действия $\varkappa$ :

$$
\varkappa=\frac{\pi \varepsilon}{2} n, \quad n=0,1,2, \ldots
$$

Значение $n=0$ отвечает случаю, когда $\left(W^{*} \Omega, v(x) W^{*} \Omega\right)=0$, т.е. солитон отсутствует. Это - единственное значение, которое сохраняет заряд $\Lambda$ (2.18) при преобразовании $(8.10),(8.11)$. При стандартном квантовании уравнения КдФ бозонное гильбертово пространство соответствует нулевому зарядовому сектору фермионного пространства Фока $\mathscr{H}$ в нашем подходе. В этом секторе преобразование (8.12) с нетривиальньм значением $n$ в (8.15) не сушествует. Это доказьвает, что солитонное состояние $W^{*} \Omega$ существует только как результат процедуры фермионизации.

\section{9. Квантование модифицированного уравнения КдФ}

Модифицированное уравнение Кортевега-де Фриза (мКд $\Phi)$

$$
v_{t}=\partial_{x}\left(g v^{3}-\frac{v_{x x}}{2}\right)
$$

на вешественную функцию $v(t, x)$ представляет собой другой хорошо известньй пример вполне интегрируемого дифференциального уравнения. Если $v(x)$ - гладкая вещественная функция, которая достаточно быстро убывает, когда $|x| \rightarrow \infty$, то метод обратной задачи рассеяния (МОЗР) (см. [29], [30] и приведенные там ссылки) применим к (9.1). Постоянная $g$ в этом уравнении - произвольньй вещественньй параметр, и свойства решений сушественно зависят от его знака. В частности, солитонные решения сушествуют, только если $g<0$.

Уравнение мКдФ - гамильтонова система по отношению к скобке (1.4), так что оно может быть записано в виде $v_{t}=-\left\{H_{\mathrm{mKdV}}, v\right\}$ с гамильтонианом

$$
H_{\mathrm{mKdV}}=\frac{1}{4} \int d x\left(g v^{4}(x)+v_{x}^{2}(x)\right)
$$

Опять реализуя $v(x)$ как в (2.11), мы выбираем квантовый гамильтониан как упорядоченное по бозонам выражение (9.2),

$$
H_{\mathrm{mKdV}}=\frac{1}{4} \int d x \vdots g v^{4}(x)+v_{x}^{2}(x) \vdots
$$


Тогда благодаря (6.24) мы получаем

$$
H_{\mathrm{mKdV}}=g H_{3}+\frac{1-g \varepsilon^{2}}{4} \int d x \vdots v_{x}^{2}:(x),
$$

где было использовано (6.24) для $n=3$. Таким образом, по аналогии со случаем Кд $\Phi$ (см. раздел 8) наиболее сингулярная часть гамильтониана (9.3), которая была четвертого порядка по отношению к бозонньм операторам, оказалась только второго порядка по отношению к фермионным. Принимая во внимание (8.4), мы получаем, что оба слагаемых в (9.4) билинейны либо по фермионным, либо по бозонным операторам и имеют диагональный вид. Соответственно, оба эти слагаемые суть хорошо определенные самосопряженные операторы в $\mathscr{H}$, и в рамках нашей процедуры квантования нет нужды ни в какой регуляризации. В частности, по (2.24) и (6.23)

$$
H_{\mathrm{mKdV}} \Omega=0
$$

а по (6.24) и (8.4) гамильтониан (9.4) положительно определен, когда $\varepsilon^{-2} \geqslant g \geqslant 0$.

Понятно, что временная эволющия, задаваемая гамильтонианом (9.3),

$$
v_{t}=i\left[H_{\mathrm{mKdV}}, v\right] \equiv \partial_{x}\left(g \vdots v^{3}:-\frac{v_{x x}}{2}\right)
$$

есть в точности квантовая версия уравнения (9.1), нормально упорядоченная по отношению к бозонньм операторам. Благодаря (6.8) и (6.15) слагаемое $v^{3}$ можно исключить, и мы получаем квантовую билинейную в терминах фермионных полей форму уравнения мКдФ:

$$
\frac{\partial}{\partial t}: \psi^{*} \psi:(x)=\frac{\partial}{\partial x}\left(-\frac{3 g \varepsilon^{2}}{4} D^{2}\left(: \psi^{*} \cdot \psi:\right)(x)+\frac{g \varepsilon^{2}-2}{4} \frac{\partial^{2}}{\partial x^{2}}: \psi^{*} \psi:(x)\right),
$$

которая может рассматриваться как квантовая форма Хироты данного уравнения (cp. $(8.7))$.

Для того чтобы вывести временную эволюцию фермионного поля $\psi$, перепишем второе слагаемое в (9.4) посредством (2.33) в смысле фермионного нормального упорядочения:

$$
H_{\mathrm{mKdV}}=\frac{5 g+\varepsilon^{-2}}{6} H_{3}+\frac{\varepsilon^{-2}-g}{2} \int d x: \psi_{x}^{*} \psi^{*} \psi_{x} \psi:(x)
$$

Тогда по (6.35) временная эволюция фермионного поля, $\psi_{t}=i\left[H_{\mathrm{mKdV}}, \psi\right]$, дается уравнением

$$
\psi_{t}(x)=-\frac{5 g \varepsilon^{2}+1}{6} \psi_{x x x}(x)+\frac{g \varepsilon^{2}-1}{2 i \varepsilon}: v_{x x} \psi:(x),
$$

которое, конечно, нелинейно при $g \neq \varepsilon^{-2}$. Подобно разделу 8 можно показать, что в $\mathscr{H}$ при $g<0$ существует односолитонное состояние. 


\section{0. Заключительные замечания}

В разделе 6 мы вьвели иерархию нелинейных интегрируемых и явно решаемых эволюционных уравнений для бозонного поля $v(x)$, реализованного в качестве композиции фермионных полей - тока. По (6.10) это означает, что в качестве динамической переменной была выбрана $F_{0}(x)$. Однако замкнутая подалгебра коммутационных соотношений (6.19)-(6.21) также дается $F_{0}(x)$ и $F_{1}(x)$. Более того, линейная комбинация

$$
\widetilde{F}(x)=F_{1}(x)+a \partial_{x} F_{0}(x)
$$

с вещественным постоянным коэффициентом $a$ также обладает замкнутым коммутационным соотношением

$$
\left[\widetilde{F}(x), \widetilde{F}\left(x^{\prime}\right)\right]=i\left\{\widetilde{F}(x)+\widetilde{F}\left(x^{\prime}\right)\right\} \delta^{\prime}\left(x-x^{\prime}\right)-i\left(a^{2}+\frac{\varepsilon^{2}}{12}\right) \delta^{\prime \prime \prime}\left(x-x^{\prime}\right)
$$

как следует из (6.19)-(6.21). Это означает, что $\widetilde{F}(x)$ дает другой возможный выбор динамической переменной. В [6] мы доказали, что бездисперсное уравнение КдФ разрешимо и в этом случае, хотя, в отличие от сказанного выше, в этом случае линейно эволюционировал ток $v(x)$. Естественно предполагать, что то же самое свойство справедливо и для всей иерархии (6.31), порождаемой квантовой версией (10.2) скобки Магри (1.5).

В разделах 8 и 9 было показано, что процедура фермионизации сушественно понижает операторные степени соответствуюших гамильтонианов, сводит их к билинейным и, таким образом, позволяет исследовать их. Достаточно удивительным является тот факт, что “фермионные” свойства интегрируемых моделей можно обнаружить уже на классическом уровне. Действительно, для $N$-солитонного решения уравнения КдФ с параметрами $\varkappa_{1}, \ldots, \varkappa_{N}$ (ср. (8.9)) посредством МОЗР доказывается [29], [30], что эти параметры никогда не совпадают:

$$
\varkappa_{i} \neq \varkappa_{j}, \quad i \neq j .
$$

Другими словами, хотя переменные $\varkappa_{1}, \ldots, \varkappa_{N}$ суть переменные действия, соответствуюшее фазовое пространство не плоско. Понятно, что условие (10.3) является условием типа принципа Паули, которьй специфичен для фермионов, т.е. для квантового случая. "Фермионные" свойства классических солитонов также наблюдались в [45] и [46] при исследовании сингулярных решений.

Сравнение уравнений (2.3) и (3.27) демонстрирует другое специфическое свойство фермионных полей: первое уравнение представляет функции $\psi_{ \pm}$как суммы функций, аналитических в верхней и нижней полуплоскостях, в то время как второе уравнение есть не что иное, как разложение тех же самых функций в произведение множителей, обладающих теми же свойствами аналитичности. Связь между этими двумя задачами Римана-Гильберта дается процедурой бозонизации. Можно надеяться, что эта интерпретация даст наводяшее соображение для расширения процедуры бозонизации на случай массивных полей.

Автор благодарит С. П. Новикова за полезные обсуждения. 


\section{СПИСОК ЛИТЕРАТУРЫ}

[1] А. Вайтман. Проблемы в релятивистской динамике квантованных полей. М.: Наука, 1968.

[2] S. Coleman. Quantum sine-Gordon equation as the massive Thirring model // Phys. Rev. D. 1975. V. 11. P. 2088-2097.

[3] S. Mandelstam. Soliton operators for the quantized sine-Gordon equation // Phys. Rev. D. 1975. V. 11. P. 3026-3030.

[4] А. К. Погребков, В. Н. Сушко. Квантование $(\sin \varphi)_{2}$-взаимодействия в терминах фермионных переменных // ТМФ. 1975. Т. 24. С. 425-429.

[5] А. К. Погребков, В.Н.Сушко. Квантовые солитоны и их связь с фермионными полями при $(\sin \varphi)_{2}$-взаимодействии // ТМФ. 1976. Т. 26. С. 419-423.

[6] А. К. Погребков. О квантовании уравнения КдФ // ТМФ. 2001. Т. 129. С. 333-344.

[7] E. Date, M. Jimbo, M. Kashiwara, T. Miwa. Transformation groups for soliton equations. III. Operator approach to the Kadomtsev-Petviashvili equation // J. Phys. Soc. Japan. 1981. V. 50. P. 3806-3812; Transformation groups for soliton equations. IV. A new hierarchy of solution equations of KP type // J. Phys. Soc. Japan. 1981. V. 50. P. 3813-3818; // Phys. D. 1981/82. V. 4. P. 343-365.

[8] T. Miwa, M. Jimbo, E. Date. Solitons: Differential Equations, Symmetries and Infinite Dimensional Algebras. Cambridge: Cambridge Univ. Press, 2000.

[9] J. A. Swieca. Solitons and confinement // Fortschr. Phys. 1977. V. 25. P. 303-326.

[10] L. K. Hadjiivanov, S. G. Mikhov, D. T. Stoyanov. On the massless scalar field in two space-time dimensions and the Thirring model // J. Phys. A. 1979. V. 12. P. 119-139.

[11] A. L. Carey, S. N. M. Ruijsenaars, J. D. Wright. The massless Thirring model: positivity of Klaiber's $n$ point functions // Comm. Math. Phys. 1985. V. 99. P. 347-364.

[12] G. Morchio, D. Pierotti, F. Strocchi. Infrared and vacuum structure in two-dimensional local quantum field theory models. The massless scalar field // J. Math. Phys. 1990. V. 31. P. 1467-1477; Infrared and vacuum structure in two-dimensional local quantum field theory models. 2. Fermion bosonization // J. Math. Phys. 1992. V. 33. P. 777-790.

[13] Ф. Коломо, А. Г. Изергин, В.Е. Корепин, В. Тогнетти. Температурные корреляционные функции в $X X O$-цепочке Гейзенберга. I // ТМФ. 1993. Т. 94. С. 19-51.

[14] Н. М. Боголюбов, А. Г. Изергин, В. Е. Корепин. Корреляционные функции интегрируемых систем и квантовьй метод обратной задачи. М.: Наука, 1992.

[15] Н. А. Славнов. Сокращение дуалшных полей в свободнофермионных моделях с тригонометрической $R$-матрицей // ТМФ. 1996. Т. 108. №2 2. С. 179-192.

[16] E. Witten. Nonabelian bosonization in two dimensions // Comm. Math. Phys. 1984. V. 92. P. $455-472$.

[17] M. Wakimoto. Fock representations of the affine Lie algebra $A_{1}^{(1)} / /$ Comm. Math. Phys. 1986. V. 104. P. 605-609.

[18] V.S. Dotsenko, V.A. Fateev. Conformal algebra and multipoint correlation functions in 2D statistical models // Nuclear Phys. B. 1984. V. 240. P. 312-348.

[19] T. Eguchi, H. Ooguri. Chiral bosonization on a Riemann surface // Phys. Lett. B. 1987. V. 187. P. 127-134.

[20] O. F. Hernández. Feigin-Fuchs bosonization of Lykken parafermions and SU $(1,1)$ KacMoody algebras // Phys. Lett. B. 1989. V. 233. P. 355-358.

[21] A. Gerasimov, A. Morozov, M. Olshanetsky, A. Marshakov, S. Shatashvili. Wess-Zumino-Witten model as a theory of free fields // Internat. J. Modern Phys. A. 1990. V. 5. P. 2495-2589.

[22] P. H. Damgaard, H. B. Nielsen, R. Sollacher. Smooth bosonization: the Cheshire cat revisited // Nuclear Phys. B. 1992. V. 385. P. 227-250.

[23] V. G. Kac. Infinite-Dimensional Lie Algebras. Cambridge: Cambridge Univ. Press, 1990.

[24] B. Klaiber. The Thirring model // Quantum Theory and Statistical Physics / ed. A. O. Barut and W.E. Brittin. New York: Gordon \& Breach, 1968. P. 141-176. (Lectures in Theoret. Phys. V.10A.) 
[25] J. H. Lowenstein, J. A. Swieca. Quantum electrodynamics in two dimensions // Ann. Physics. 1971. V. 68. P. 172-195.

[26] N. Ilieva, W. Thirring. Do anyons solve Heisenberg's Urgleichung in one dimension? // Eur. Phys. J. C Part. Fields. 1999. V. 6. P. 705-714.

[27] Н. Илиева, В. Тирринг. Анионы и дуальность Бозе-Ферми в модели Тирринга при конечных температурах // ТМФ. 1999. Т. 121. C. 40-65.

[28] C.S. Gardner, J.M. Greene, M.D. Kruskal, R. M. Miura. Method for solving the Korteweg-De Vries equation // Phys. Rev. Lett. 1967. V. 19. P. 1095-1097.

[29] В.Е. Захаров, С. В. Манаков, С.П. Новиков, Л. П. Питаевский. Теория солитонов: Метод обратной задачи. М.: Наука, 1980.

[30] Ф. Калоджеро, А. Дегасперис. Спектральные преобразования и солитоны. Методы решения и иследования нелинейных эволюционных уравнений. М.: Мир, 1985.

[31] C. Gardner. Korteweg-de Vries equation and generalizations. IV. Korteweg-de Vries equation as a hamiltonian system // J. Math. Phys. 1971. V. 12. P. 1548-1551.

[32] В.Е. Захаров, Л. Д. Фаддеев. Уравнение Кортевега-де Фриса - вполне интегрируемая гамильтонова система // Функц. анализ и его прил. 1971. Т. 5. № 4. С. 18-27.

[33] F. Magri. A geometrical approach to the nonlinear solvable equations // Nonlinear Evolution Equations and Dynamical Systems / ed. M. Boiti, F. Pempinelli, G. Soliani. Berlin: Springer-Verlag, 1980. P. 233-263. (Lecture Notes in Phys. V. 120.)

[34] P. B. Wiegmann, A. Zabrodin. Conformal maps and integrable hierarchies // Comm. Math. Phys. 2000. V. 213. P. 523-538.

[35] J. Barcelos-Neto, A. Constandache, A. Das. Dispersionless fermionic KdV // Phys. Lett. A. 2000. V. 268. P. 342-351.

[36] V.V. Bazhanov, S. L. Lukyanov, A. B. Zamolodchikov. Integrable structure of conformal field theory, quantum KdV theory and thermodynamic Bethe ansatz // Comm. Math. Phys. 1996. V. 177. P. 381-398; Integrable structure of conformal field theory. II. $Q$-operator and DDV equation // Comm. Math. Phys. 1997. V. 190. P. 247-278; Integrable structure of conformal field theory. III. The Yang-Baxter relation // Comm. Math. Phys. 1999. V. 200. P. 297-324.

[37] P. Di Francesco, P. Mathieu, D. Sénéchal. Integrability of the quantum KdV equation at $C=-2 / /$ Modern Phys. Lett. A. 1992. V. 7. P. 701-707.

[38] I. Krichever. The dispersionless Lax equations and topological minimal models // Comm. Math. Phys. 1992. V. 143. P. 415-429.

[39] K. Takasaki, T. Takebe. Integrable hierarchies and dispersionless limit // Rev. Math. Phys. 1995. V. 7. P. 743-808.

[40] A. Boyarsky, A. Marshakov, O. Ruchayskiy, P. Wiegmann, A. Zabrodin. Associativity equations in dispersionless integrable hierarchies // Phys. Lett. B. 2001. V. 515. P. 483-492.

[41] A. K. Pogrebkov, M. C. Prati. On bosonization of free massless fermions. I. Periodic case // Nuovo Cimento A. 1994. V. 107. P. 1315-1323; On bosonization of free massless fermions. II. Case of infinite line // Nuovo Cimento A. 1996. V. 109. P. 9-17.

[42] Ф. А. Березин. Метод вторичного квантования. М.: Наука, 1965.

[43] М. Рид, Б. Саймон. Методы современной математической физики. 1. Функциональный анализ. М.: Мир, 1977.

[44] A. K. Pogrebkov. Hierarchy of quantum explicitly solvable and integrable models // nlin.SI/0202043, 2002.

[45] В. А. Аркадьев, А. К. Погребков, М. К. Поливанов. Сингулярные решения уравнения КдВ и метод обратной задачи // Зап. научн. сем. ЛОМИ. 1984. Т. 133. С. 17-37.

[46] А. К. Погребков, М. К. Поливанов. О взаимодействии частиц и полей в классической теории // Проблемы физики элементарных частиц и атомного ядра (ЭЧАЯ). 1983. T. 14. C. $1073-1091$. 